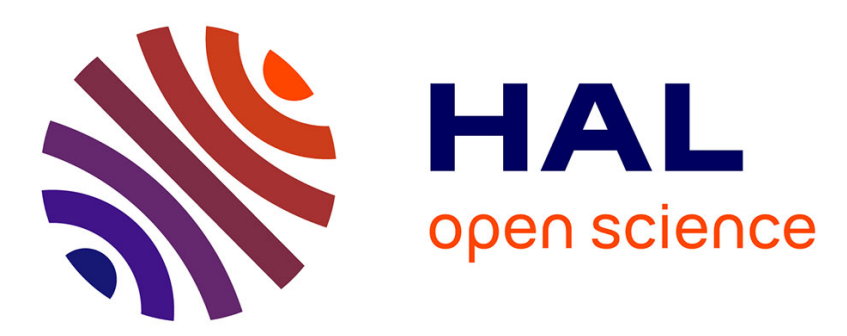

\title{
Hamiltonian gyrofluid reductions of gyrokinetic equations
}

\author{
Emanuele Tassi
}

\section{To cite this version:}

Emanuele Tassi. Hamiltonian gyrofluid reductions of gyrokinetic equations. Journal of Physics A: Mathematical and Theoretical, 2019, pp.465501. hal-02361125

\section{HAL Id: hal-02361125 \\ https://hal.science/hal-02361125}

Submitted on 13 Nov 2019

HAL is a multi-disciplinary open access archive for the deposit and dissemination of scientific research documents, whether they are published or not. The documents may come from teaching and research institutions in France or abroad, or from public or private research centers.
L'archive ouverte pluridisciplinaire HAL, est destinée au dépôt et à la diffusion de documents scientifiques de niveau recherche, publiés ou non, émanant des établissements d'enseignement et de recherche français ou étrangers, des laboratoires publics ou privés. 


\title{
Hamiltonian gyrofluid reductions of gyrokinetic equations
}

\author{
E. Tassi ${ }^{1}$ \\ ${ }^{1}$ Université Côte d'Azur, CNRS, Observatoire de la Côte d'Azur, Laboratoire J. L. \\ Lagrange, Boulevard de l'Observatoire, CS 34229, 06304 Nice Cedex 4, France
}

\begin{abstract}
We provide a general framework for deriving Hamiltonian electromagnetic gyrofluid models from a Hamiltonian system of gyrokinetic equations. The presented procedure permits to derive gyrofluid models for an arbitrary number of moments with respect to the velocity coordinate parallel to an equilibrium magnetic field. The resulting gyrofluid models account, in particular, for finite Larmor radius effects, equilibrium temperature anisotropies and fluctuations of the magnetic field in the direction parallel to the equilibrium magnetic field, thus generalizing Hamiltonian gyrofluid models previously presented in the literature. The Hamiltonian reduction procedure leading from the parent gyrokinetic model to the gyrofluid models is formulated in two stages. In the first step, after having shown that the parent gyrokinetic system indeed posseses a Hamiltonian structure, a Hamiltonian system is derived, by means of a Poisson sub-algebra argument, which describes the evolution of the perturbation of the gyrocenter distribution function, averaged with respect to the magnetic moment coordinate. The second stage brings from the latter model to the gyrofluid models by means of a closure relation, applicable at an arbitrary order in the moment hierarchy, which guarantees the preservation of a Hamiltonian structure. Casimir invariants of the noncanonical Poisson brackets of the gyrofluid models are provided. It is also shown how, in the two-dimensional limit, the gyrofluid model equations can be cast in the form of advection equations for Lagrangian invariants transported by generalized incompressible velocity fields, thus extending results obtained for previous Hamiltonian gyrofluid and drift-fluid models. The Hamiltonian reduction procedure is applied to derive a five-field gyrofluid model evolving the first two moments for the electron species and the first three moments for the ion species. The Casimir invariants and the Lagrangian advection formulation are provided explicitly for the five-field model. Remarks concerning possible variants of the procedure are discussed. As an example, it is shown how, by means of a variant of the procedure, it is possible to derive an isothermal two-field model for kinetic Alfvén waves including equilibrium electron temperature anisotropy effects.
\end{abstract}




\section{Introduction}

Reduction from kinetic to fluid systems is a frequently adopted operation for modelling many-particle systems, such as gases and plasmas, that can be treated as continuous media. Indeed, fluid systems possess the advantage that the corresponding dynamical variables, i.e. the fluid moments, depend on a reduced number of coordinates, with respect to the dynamical variables of the original kinetic models, consisting of distribution functions. This makes fluid models in general more amenable to analytical treatments, with respect to kinetic models, and also less demanding in terms of computational resources required for numerical integration. Clearly, due to the loss of information occurring in the reduction procedure, the drawback of fluid models is their inability in describing, for instance, phenomena such as wave-particle interactions in plasmas. When an accurate description of such phenomena is not prior, however, fluid models provide an effective alternative to the more complete kinetic models. In most cases, the starting point in reductions from kinetic to fluid systems is a kinetic model (e.g. Vlasov-Maxwell or Vlasov-Poisson) which contains partial differential equations $\partial_{t} f=F$ evolving one-particle distribution functions of the form $f\left(\mathrm{x}_{1}, \cdots, \mathrm{x}_{m}, \mathrm{v}_{1}, \cdots \mathrm{v}_{n}, t\right)$, with $1 \leq m, n \leq 3$. The coordinates $\left(\mathrm{x}_{1}, \cdots, \mathrm{x}_{m}\right) \in \mathbb{R}^{m}$ indicate a position in space, whereas $\left(\mathrm{v}_{1}, \cdots, \mathrm{v}_{n}\right) \in \mathbb{R}^{n}$ indicate a set of coordinates in velocity space and $t \in \mathbb{R}$ indicates time. The evolution operator $F$ is, in general an integro-differential operator depending on $\mathrm{x}_{1}, \cdots, \mathrm{x}_{m}, \mathrm{v}_{1}, \cdots, \mathrm{v}_{n}, t$, on $f$ and, possibly, on further dynamical variables that might be present in the system (e.g. electromagnetic fields in the case of the Vlasov-Maxwell system). The value of the function $f\left(\mathrm{x}_{1}, \cdots, \mathrm{x}_{m}, \mathrm{v}_{1}, \cdots \mathrm{v}_{n}, t\right)$ represents the probability density of finding a particle at position $\left(\mathrm{v}_{1}, \cdots, \mathrm{v}_{n}\right)$ in the velocity space, with spatial coordinates $\left(\mathrm{x}_{1}, \cdots, \mathrm{x}_{m}\right)$ at time $t$. The reduction consists of deriving, from the kinetic model, a fluid model evolving in time a finite set $\mathbb{P}=\left\{f_{i_{1}, \cdots, i_{n}}\left(\mathrm{x}_{1}, \cdots, \mathrm{x}_{m}, t\right): 0 \leq\right.$ $i_{j} \leq \mathcal{N}_{j}$ for $\left.j=1, \cdots, n\right\}$, of (fluid) moments $f_{i_{1}, \cdots, i_{n}}$, where $\mathcal{N}_{1}, \cdots, \mathcal{N}_{n}$ are nonnegative integers determining the number of moments retained in the fluid model. The fluid moments thus only depend on a reduced number of coordinates (spatial 
coordinates and time) and are typically obtained by integrating, in velocity space, the distribution function multiplied by a polynomial in the velocity coordinates. Upon choosing a basis of the form $\mathcal{P}_{1}\left(\mathrm{v}_{1}\right), \cdots, \mathcal{P}_{n}\left(\mathrm{v}_{n}\right)$ for the polynomials in velocity space, the generic moment $f_{i_{1}, \cdots, i_{n}}\left(\mathrm{x}_{1}, \cdots, \mathrm{x}_{m}, t\right)$, with respect to the chosen basis, can be defined as $f_{i_{1}, \cdots, i_{n}}\left(\mathrm{x}_{1}, \cdots, \mathrm{x}_{m}, t\right)=\int d \mathrm{v} \prod_{j=1}^{n} \mathcal{P}_{j}{ }^{i_{j}}\left(\mathrm{v}_{j}\right) f\left(\mathrm{x}_{1}, \cdots, \mathrm{x}_{m}, \mathrm{v}_{1}, \cdots, \mathrm{v}_{n}, t\right)$, where $d \mathrm{v}$ indicates the volume element in velocity space. One of the main difficulties in the reduction procedure, as is well known, lies in the fact that the evolution equation for a moment $f_{i_{1}, \cdots, i_{n}} \in \mathbb{P}$, (which, according to the ordinary procedure, is obtained from the kinetic equation $\partial_{t} f=F$ upon multiplying both sides of the equation by $\prod_{j=1}^{n} \mathcal{P}_{j}{ }^{i_{j}}\left(\mathrm{v}_{j}\right)$ and integrating over velocity space), is of the form $\partial_{t} f_{i_{1}, \cdots, i_{n}}=G_{i_{1}, \cdots, i_{n}}$, where the evolution operator $G_{i_{1}, \cdots, i_{n}}$ can depend also on a (possibly infinite) number of moments $f_{j_{1}, \cdots, j_{n}}$ not belonging to $\mathbb{P}$ because, for some $k, j_{k}>\mathcal{N}_{k}$.

This leads to a closure problem, which consists of imposing, for all moments $f_{j_{1}, \cdots, j_{n}}$ appearing in the evolution operators $G_{i_{1}, \cdots, i_{n}}$ but not belonging to $\mathbb{P}$, relations of the form $f_{j_{1}, \cdots, j_{n}}=\mathfrak{P}_{j_{1}, \cdots, j_{n}}$, where $\mathfrak{P}_{j_{1}, \cdots, j_{n}}$ is a set of operators depending only on the moments belonging to $\mathbb{P}$. In this way, the evolution operators $G_{i_{1}, \cdots, i_{n}}$ only depend on the moments $f_{i_{1}, \cdots, i_{n}} \in \mathbb{P}$ and the resulting system is closed. Clearly, the choice of the closure operators $\mathfrak{P}_{j_{1}, \cdots, j_{n}}$ is crucial in determining the dynamical properties of the fluid system.

In the physics of strongly magnetized plasmas, one of the most commonly adopted kinetic theories is the so called gyrokinetic theory (see, e.g. Refs.[1, 2]). A large number of gyrokinetic models have been derived, with various assumptions depending on specific applications. The gyrokinetic model considered in this manuscript, which was derived in Ref. [3], belongs to the class of so-called $\delta f$ gyrokinetic models, which assume the distribution functions to be close to equilibrium ones. The model describes the evolution of the first order (according to a prescribed ordering) perturbations of the equilibrium distribution functions for each particle species. In particular, the model can be formulated as a system of evolution equations for the perturbations of the gyrocenter 
distribution functions $\widetilde{f}_{s}\left(x_{1}, x_{2}, x_{3}, v_{\|}, \mu_{0 s}, t\right)$, corresponding to the case $m=3, n=2$ of the general situation described above. Such evolution equations are valid only to a certain order (for instance they neglect terms which are higher than quadratic in the small perturbations). The coordinates in velocity space are denoted as $v_{\|}$and $\mu_{s}$ and represent the gyrocenter velocity coordinate along the equilibrium magnetic field and the adiabatic invariant related to the species $s$. In principle, space and velocity coordinates of the gyrocenter distribution functions are obtained by means of a near-identity transformation from the guiding-center coordinates (see, e.g. Ref. [4]). However, for the perturbation $\tilde{f}_{s}$, it is required to consider, for each coordinate, only the leading order term in this transformation. In particular, only the leading order term $\mu_{0 s}$, corresponding to the magnetic moment in the presence of the unperturbed magnetic field, contributes in the expression for the adiabatic invariant $\mu_{s}$. The higher order terms indeed yield negligible contributions, in the asymptotic regime of validity of the model. $\delta f$ gyrokinetic equations are evolution equations of the form $\partial_{t} \widetilde{f}_{s}=F_{s}$, where $F_{s}$ are operators, associated with the particle species $s$, that depend on $\widetilde{f}_{s}$ (and possibly on electromagnetic fields, if these are independent dynamical variables of the system, satisfying their own evolution equations). A fluid reduction problem, as above described, emerges, when one intends to obtain, from the gyrokinetic equations $\partial_{t} \widetilde{f}_{s}=F_{s}$, a gyrofluid [4] system, that is a closed system of equations of the form $\partial_{t} P_{i_{1}, i_{2 s}}=G_{i_{1}, i_{2} 2_{s}}$, with $P_{i_{1}, i_{2}}\left(\mathrm{x}_{1}, \mathrm{x}_{2}, \mathrm{x}_{3}, t\right)=\int d \mathrm{v} \mathcal{P}_{1}^{i_{1}}\left(v_{\|}\right) \mathcal{P}_{2}^{i_{2}}\left(\mu_{s}\right) \widetilde{f}_{s}\left(\mathrm{x}_{1}, \mathrm{x}_{2}, \mathrm{x}_{3}, v_{\|}, \mu_{s}, t\right)$, for $0 \leq i_{1} \leq \mathcal{N}_{1_{s}}$, $0 \leq i_{2} \leq \mathcal{N}_{2_{s}}$, with non-negative integers $\mathcal{N}_{1_{s}}, \mathcal{N}_{2_{s}}$. We remark that, due to the presence of the so called gyroaverage operators, which involve all powers of the magnetic moment coordinate $\mu_{s}$ in the parent gyrokinetic system, the evolution operators $G_{i_{1}, i_{2}}$ depend on an infinity of moments with respect to the $\mu_{s}$ coordinate, for a given basis.

In the plasma physics literature, various approaches to the closure problem of gyrofluid models (also outside the $\delta f$ limit) have been adopted. In Refs. [5, 6, 7, 8, 9], electrostatic and electromagnetic gyrofluid models have been derived, adopting closure relations such that the dispersion relations obtained from the linearized gyrofluid 
models agree with those of the linearized gyrokinetic parent equations. Thanks to this approach, the phenomenon of Landau damping, which requires a kinetic description, can be modelled, at least in the linear phase, also by means of gyrofluid models. In Ref. [4], although the closure problem is not explicitly addressed, an expansion of the perturbation of the distribution functions is assumed, which allows to determine moments involving gyroaverage operators. Closure relations guaranteeing energy conservation in gyrofluid models are adopted in Refs. [10, 11, 12]. In recent years, a number of gyrofluid models were derived $[13,14,15,16]$, for which, in addition to energy conservation, the existence of a noncanonical Hamiltonian structure was proved. This guarantees that, in the non-dissipative limit, the intrinsic Hamiltonian character of the original gyrokinetic dynamics is preserved. This property also turned out to be useful for deriving invariant quantities (Casimir invariants), carrying out stability analyses [16] and interpreting numerical simulations of magnetic reconnection by means of gyrofluid models $[17,18,15]$. In the above References $[13,14,15,16]$, the Hamiltonian structure of the gyrofluid models was, however, found a posteriori, once the model equations were derived by imposing a closure relation guaranteeing energy conservation. Also, all of the models described in such references, apply to plasmas with $\beta \ll 1$ (where $\beta$ indicates the ratio between the internal and the magnetic pressure in the plasma), thus neglecting perturbations of the magnetic field along the direction of the equilibrium magnetic field (we point out, however, the existence of a model, described in Ref. [19], accounting for such perturbations, but for which no Hamiltonian structure is known). Equilibrium temperature anisotropies were also neglected in those models, assuming that the equilibrium distribution function of the parent gyrokinetic model was a Maxwellian distribution.

The purpose of this paper is to provide a general framework for deriving gyrofluid models from a Hamiltonian gyrokinetic system, in such a way that the resulting gyrofluid models also possess a Hamiltonian structure. The Hamiltonian reduction that we present, permits to derive Hamiltonian gyrofluid models evolving an arbitrary number 
of moments with respect to the (normalized) $v_{\|}$coordinate. A convenient choice of the basis of polynomials adopted for defining the moments is the customary one, consisting of Hermite polynomials for the (normalized) parallel velocity and of Laguerre polynomials for the (normalized) magnetic moment. The gyrofluid models derived with this procedure are valid also for values of $\beta \sim 1$ and account for equilibrium temperature anisotropies, originating from the choice of a bi-Maxwellian equilibrium distribution function in the parent gyrokinetic model. Therefore, the Hamiltonian gyrofluid models derived with our procedure, permit to extend the Hamiltonian gyrofluid models present in the literature, by the inclusion of parallel magnetic perturbations and equilibrium temperature anisotropies. These two aspects can be particularly relevant in view of the application of gyrofluid models for the investigation of phenomena relevant to space plasmas. In this respect, the reduced gyrofluid models presented here, could act as a tool, complementary to more realistic (but also more complex) kinetic and fluid models, for the investigation of basic phenomena relevant for the solar wind and the magnetosphere. These could include, for instance, instabilities induced by equilibrium temperature anisotropies, kinetic Alfvén wave turbulence and collisionless reconnection in asymmetric configurations. A complete description of space plasma phenomena would of course require much more complex models accounting for, among other aspects, inhomogeneous equilibria and more realistic boundary conditions. Such description lies outside the scope of the present reduced gyrofluid models.

It is important to point out that the results obtained from the different approaches to closures mentioned above, are not necessarily in conflict among each other. In particular, results obtained from our procedure, which emphasizes the importance of a Hamiltonian structure, could be compatible with those obtained from more traditional approaches based on kinetic closures or energy conservation. The Hamiltonian derivation, nevertheless, provides additional information on the model and puts it on more solid ground. From this point of view, the case where higher-order closures are imposed on both coordinates in velocity space (i.e. $\mathcal{N}_{1_{s}} \geq 2$ and $\mathcal{N}_{2_{s}} \geq 2$ ) and for which 
a Hamiltonian theory is, at the moment, essentially absent, represents an interesting and challenging problem, also with regard to the comparison between the results obtained by means of the different approaches. Moreover, as above mentioned, the present procedure permits to derive gyrofluid models in their non-dissipative limit. Dissipative terms, possibly obtained from the linear kinetic theory, can of course be added a posteriori.

The structure of the paper is the following. In Sec. 2 we introduce and review the main physical ingredients of the parent gyrokinetic model (which is taken from Ref. [3]) in the non-dissipative limit. In Sec. 3 we show that such gyrokinetic model possesses, as required, a Hamiltonian structure. In Sec. 4 we present the Hamiltonian reduction to gyrofluid models, which is formulated in two stages. The first one brings from the Hamiltonian parent gyrokinetic model to a reduced Hamiltonian model, still of kinetic nature, which evolves the perturbation of the gyrocenter distribution function averaged with respect to the magnetic moment coordinate. The parent gyrokinetic model evolves the perturbation of the distribution function denoted, for the species $s$, as $\widetilde{f}_{s}\left(x, y, z, v_{\|}, \mu_{0 s}, t\right)$ (actually, an alternative variable $\widetilde{g}_{s}\left(x, y, z, v_{\|}, \mu_{0 s}, t\right)$, including perpendicular magnetic perturbations, is most often used in the paper). The model obtained after the first stage of the reduction, on the other hand, evolves the averaged distribution function $f_{0_{s}}\left(x, y, z, v_{\|}, t\right)$, thus reducing the dimension of the velocity coordinate space from $n=2$ to $n=1$. The preservation of the Hamiltonian character is guaranteed, in this stage, by the property that functionals of $f_{0_{s}}$ form a sub-algebra with respect to the Poisson bracket of the parent gyrokinetic model. We remark that the first stage of the reduction implies that all moments of $\widetilde{f}_{s}\left(x, y, z, v_{\|}, \mu_{0 s}, t\right)$ of order $i_{2} \geq 1$ with respect to Laguerre polynomials in a normalized $\mu_{0 s}$ coordinate are set equal to zero. Physically, this corresponds to setting equal to zero all the moments involving powers of the velocity component perpendicular to the equilibrium magnetic field, such as, for instance, the perpendicular temperature and heat flux. This solves (although rather brutally) the above mentioned closure problem with respect to moments in the magnetic moment coordinate. The second stage leads from the reduced kinetic model 
obtained from the first stage, to the actual Hamiltonian gyrofluid models, evolving moments $f_{0 i_{1 s}}(x, y, z, t)$ of $f_{0_{s}}\left(x, y, z, v_{\|}, t\right)$, with respect to a basis of normalized Hermite polynomials in the velocity coordinate $v_{\|}$. The procedure of the second stage extends results obtained in Ref. [20] and leads to a family of Hamiltonian gyrofluid models for an arbitrary number $\mathcal{N}_{s}+1$ of moments, for each species. Casimir invariants of the resulting models are presented and it is shown that, in the limit of invariance along the direction of the equilibrium magnetic field, the model equations take the form of advection equations for Lagrangian invariants. The result of the Hamiltonian reduction is exemplified in Sec. 5, where a five-field Hamiltonian gyrofluid model for a plasma consisting of two species is constructed, following the procedure described in Sec. 4 . The Casimir invariants and the two-dimensional formulation of the model in terms of Lagrangian invariants are provided. Section 6 discusses some possible variants of the reduction procedure, that can allow to obtain further Hamiltonian gyrofluid models. A concrete example is discussed, which consists of a two-field model for kinetic Alfvén waves, including corrections due to electron inertia, finite ion Larmor radius and finite $\beta$ effects as well as equilibrium temperature anisotropy. The model does not descend directly from the reduction procedure of Sec. 4 but requires a variant of it, for it assumes an isothermal closure. We conclude in Sec. 7. At the end of the paper we also provide the Appendix A, where we discuss properties of the operators that permit to express electromagnetic fields in terms of the distribution function in the parent gyrokinetic model.

\section{Parent gyrokinetic model}

We consider as starting point the gyrokinetic system presented in Ref. [3] (and corresponding to Eqs. (C58), (C66), (C67) and (C68) of such reference) in the specific case where the plasma consists of only two species, namely electrons and (single ionized) ions. The equilibrium distribution function is taken to be a bi-Maxwellian for both species with no equilibrium drifts. In this limit, Eqs. (C58), (C66), (C67) and (C68) of 
Ref. [3] can be written (also with the help of Eq. (C60) of the same reference), as

$$
\begin{aligned}
& \frac{\partial \widetilde{g}_{s}}{\partial t}+\frac{c}{B_{0}}\left[J_{0}\left(a_{s}\right) \widetilde{\phi}-\frac{v_{\|}}{c} J_{0}\left(a_{s}\right) \widetilde{A}_{\|}+2 \frac{\mu_{0 s} B_{0}}{q_{s}} \frac{J_{1}\left(a_{s}\right)}{a_{s}} \frac{\widetilde{B}_{\|}}{B_{0}}, \widetilde{g}_{s}\right] \\
& +v_{\|} \frac{\partial}{\partial z}\left(\widetilde{g}_{s}+\frac{q_{s}}{T_{0_{\| s}}} \mathcal{F}_{0 s}\left(J_{0}\left(a_{s}\right) \widetilde{\phi}-\frac{v_{\|}}{c} J_{0}\left(a_{s}\right) \widetilde{A}_{\|}+2 \frac{\mu_{0 s} B_{0}}{q_{s}} \frac{J_{1}\left(a_{s}\right)}{a_{s}} \frac{\widetilde{B}_{\|}}{B_{0}}\right)\right)=0 \\
& \sum_{s} q_{s} \int d \mathcal{W}_{s} J_{0}\left(a_{s}\right) \widetilde{g}_{s}=\sum_{s} \frac{q_{s}^{2}}{T_{0_{\perp s}}} \int d \mathcal{W}_{s} \mathcal{F}_{0 s}\left(1-J_{0}^{2}\left(a_{s}\right)\right) \widetilde{\phi} \\
& -\sum_{s} q_{s} \int d \mathcal{W}_{s} 2 \frac{\mu_{0 s} B_{0}}{T_{0_{\perp s}}} \mathcal{F}_{0 s} J_{0}\left(a_{s}\right) \frac{J_{1}\left(a_{s}\right)}{a_{s}} \frac{\widetilde{B}_{\|}}{B_{0}}, \\
& \sum_{s} q_{s} \int d \mathcal{W}_{s} v_{\|} J_{0}\left(a_{s}\right)\left(\widetilde{g}_{s}-\frac{q_{s}}{T_{0_{\| s}}} \frac{v_{\|}}{c} \mathcal{F}_{0 s} J_{0}\left(a_{s}\right) \widetilde{A}_{\|}\right) \\
& =-\frac{c}{4 \pi} \nabla_{\perp}^{2} \widetilde{A}_{\|}+\sum_{s} \frac{q_{s}^{2}}{m_{s}} \int d \mathcal{W}_{s} \mathcal{F}_{0 s}\left(1-\frac{1}{\Theta_{s}} \frac{v_{\|}^{2}}{v_{t h}^{2}}\right)\left(1-J_{0 s}^{2}\left(a_{s}\right)\right) \frac{\widetilde{A}_{\|}}{c} \\
& \sum_{s} \frac{\beta_{\perp_{s}}}{n_{0}} \int d \mathcal{W}_{s} 2 \frac{\mu_{0 s} B_{0}}{T_{0_{\perp s}}} \frac{J_{1}\left(a_{s}\right)}{a_{s}} \widetilde{g}_{s}=-\sum_{s} \frac{\beta_{\perp_{s}}}{n_{0}} \frac{q_{s}}{T_{0_{\perp s}}} \int d \mathcal{W}_{s} 2 \frac{\mu_{0 s} B_{0}}{T_{0_{\perp s}}} \mathcal{F}_{0 s} J_{0}\left(a_{s}\right) \frac{J_{1}\left(a_{s}\right)}{a_{s}} \widetilde{\phi} \\
& -\left(2+\sum_{s} \frac{\beta_{\perp_{s}}}{n_{0}} \int d \mathcal{W}_{s} \mathcal{F}_{0 s}\left(2 \frac{\mu_{0 s} B_{0}}{T_{0_{\perp s}}} \frac{J_{1}\left(a_{s}\right)}{a_{s}}\right) \frac{\widetilde{B}_{\|}}{B_{0}}\right.
\end{aligned}
$$

Eq. (1) is a gyrokinetic equation governing the evolution of the perturbation of the generalized gyrocenter distribution function $\widetilde{g}_{s}$ (note the different notation for this function with respect to Ref. [3]), whereas Eqs. (2), (3) and (4) relate electromagnetic perturbations with the distribution functions and correspond to the quasi-neutrality relation and to Ampère's law in the direction parallel and perpendicular to the magnetic equilibrium guide field, respectively.

We formulate the system over the domain $\left\{\left(x, y, z, v_{\|}, \mu_{0 s}, t\right):-L_{x} \leq x \leq\right.$ $\left.L_{x},-L_{y} \leq y \leq L_{y},-L_{z} \leq z \leq L_{z},-\infty \leq v_{\|} \leq+\infty, \mu_{0 s} \geq 0, t \geq 0\right\}$, where $x, y, z$ are Cartesian coordinates indicating the spatial variables, $v_{\|}$is the velocity coordinate parallel to an equilibrium uniform and constant guide field of amplitude $B_{0}$ directed along the $z$ axis of the Cartesian coordinate system, $\mu_{0 s}$ is the magnetic moment of the particle of species $s$ (with $s=e$ for electrons and $s=i$ for ions) in the equilibrium, straight and homogeneous magnetic field, in the absence of electromagnetic perturbations. The coordinate $\mu_{0 s}$ is related to the perpendicular velocity coordinate $v_{\perp}$ 
by $\mu_{0 s}=m_{s} v_{\perp}^{2} /\left(2 B_{0}\right)$, with $m_{s}$ indicating the mass of the particle of species $s$. Clearly, the coordinates $\mu_{0 e}$ and $\mu_{0 i}$ are not independent but it will be convenient to use one or the other according to the dependent variable under consideration. We remark that we make use of the coordinate $\mu_{0 s}$ instead of the coordinate $v_{\perp}$ adopted in Ref. [3], for it turns out to be more practical in our case, given our choice (5) for the equilibrium distribution function. The coordinate $t$ is the time coordinate, whereas $L_{x}, L_{y}$ and $L_{z}$ are three positive constant determining the boundaries of the spatial domain. We assume that the variables depending on $x, y$, and $z$ satisfy periodic boundary conditions with respect to these coordinates. In particular, we assume that such space-dependent variables admit a Fourier series representation with respect to $x, y$ and $z$. For variables depending also on $v_{\|}$and $\mu_{0 s}$, we assume that their values decay to zero as $v_{\|} \rightarrow \pm \infty$ and also as $\mu_{0 s} \rightarrow+\infty$, for every $s$, whereas we assume that they have a smooth dependence on $x, y, z, v_{\|}$at any time $t$, at $\mu_{s}=0$, for every $s$.

As above anticipated, compared to Ref. [3], we restrict our analysis to the case where the equilibrium distribution functions $\mathcal{F}_{0 s}$ are bi-Maxwellians with no equilibrium flows. Given its order of accuracy, the model requires to make use only of the leading order term, with respect to electromagnetic perturbations or in Larmor radius expansion, of the bi-Maxwellian equilibrium distribution functions of gyrocenter particle invariants. In terms of the adopted coordinates $v_{\|}$and $\mu_{0 s}$, the explicit expressions for such distribution functions at leading order are given by

$$
\mathcal{F}_{0 s}\left(v_{\|}, \mu_{0 s}\right)=\left(\frac{m_{s}}{2 \pi}\right)^{3 / 2} \frac{n_{0}}{T_{0_{\| s}}^{1 / 2} T_{0_{\perp s}}} \mathrm{e}^{-\frac{m_{s} v_{\|}^{2}}{2 T_{0}}-\frac{\mu_{0 s} B_{0}}{T_{0}}}
$$

where $n_{0}$ is the uniform equilibrium density (assumed to be identical for both species), whereas $T_{0_{\| s}}$ and $T_{0_{\perp s}}$ are the uniform equilibrium temperatures for the $s$-th species, in the direction parallel and perpendicular to the guide field, respectively. First order corrections, due to electromagnetic perturbations, to the leading order distribution functions (5) intervene in the transformation that relates the perturbation of the gyrocenter distribution function with the perturbation of the particle distribution 
function [3].

In the system (1)-(4) the function $\widetilde{g}_{s}$ is defined by

$$
\widetilde{g}_{s}\left(x, y, z, v_{\|}, \mu_{0 s}, t\right)=\widetilde{f}_{s}\left(x, y, z, v_{\|}, \mu_{0 s}, t\right)+\frac{q_{s}}{T_{0_{\| s}}} \frac{v_{\|}}{c} \mathcal{F}_{0 s}\left(v_{\|}, \mu_{0 s}\right) J_{0}\left(a_{s}\right) \widetilde{A}_{\|}(x, y, z, t),
$$

where $\widetilde{f}_{s}$ is the perturbed distribution function for the gyrocenters of the species $s, q_{s}$ is the charge of the particle of the $s$-th species (given that we are considering single ionized ions we have $q_{e}=-e$ and $q_{i}=e$, where $e$ is the proton charge) and $c$ is the speed of light. The constants $v_{t h_{\| s}}=\sqrt{T_{0_{\| s}} / m_{s}}$, on the other hand, indicate the thermal parallel speed associated with the species $s$. The field $\widetilde{A}_{\|}$indicates the perturbation of the magnetic field in the plane perpendicular to the guide field. More precisely, up to second order terms in the perturbations, we consider a magnetic field $\mathbf{B}$ of the form

$$
\mathbf{B}(x, y, z, t)=\nabla \widetilde{A}_{\|}(x, y, z, t) \times \hat{z}+\left(B_{0}+\widetilde{B}_{\|}(x, y, z, t)\right) \hat{z}
$$

with $\widetilde{B}_{\|}$indicating, on the other hand, the perturbation along the direction of the guide field.

Resorting to the Fourier-series representation of the space-dependent variables, and upon introducing the wave numbers $k_{x}=m \pi / L_{x}, k_{y}=n \pi / L_{y}$ and $k_{z}=p \pi / L_{z}$ for $m, n, p \in \mathbb{Z}$, we can introduce the standard gyroaverage operator $J_{0}$. Given a field $f$ periodic in the spatial variables, we represent it as $f(\mathbf{x}, t)=\sum_{\mathbf{k} \in \mathscr{D}} f_{\mathbf{k}}(t) \exp (i \mathbf{k} \cdot \mathbf{x})$, where $\mathbf{k}=\left(k_{x}, k_{y}, k_{z}\right), \mathbf{x}=(x, y, z)$, and $\mathscr{D}$ is the lattice defined by $\mathscr{D}=$ $\left\{\left(m \pi / L_{x}, n \pi / L_{y}, p \pi / L_{z}\right):(m, n, p) \in \mathbb{Z}^{3}\right\}$. Clearly, in order for the system (1)-(4) to be well defined, it is required that the equations (2)-(4) permit to express $\widetilde{\phi}, \widetilde{A}_{\|}$and $\widetilde{B}_{\|}$ in terms of $\widetilde{g}_{e}$ and $\widetilde{g}_{i}$. This might in principle impose conditions on some of the Fourier components of $\widetilde{g}_{e}$ and $\widetilde{g}_{i}$. This issued is discussed in the Appendix. The gyroaveraged field $J_{0}\left(a_{s}\right) f$ is then defined as $J_{0}\left(a_{s}\right) f(\mathbf{x}, t)=\sum_{\mathbf{k} \in \mathscr{D}} J_{0}\left(a_{s}\right) f_{\mathbf{k}}(t) \exp (i \mathbf{k} \cdot \mathbf{x})$, where $J_{0}$ is the zeroth order Bessel function and

$$
a_{s}=\frac{k_{\perp} v_{\perp}}{\omega_{c s}}=\frac{k_{\perp}}{\omega_{c s}} \sqrt{\frac{2 \mu_{0 s} B_{0}}{m_{s}}},
$$

with $k_{\perp}=\sqrt{k_{x}^{2}+k_{y}^{2}}$ the perpendicular wave number and $\omega_{c s}=e B_{0} /\left(m_{s} c\right)$ the cyclotron frequency associated with the species $s$. The above definition of the gyroaverage operator 
$J_{0}$ is extended in a standard and straightforward way to the other gyroaverage operators appearing in Eqs. (1)-(4) and involving also the first order Bessel function $J_{1}$.

We complete the definitions of the quantities appearing in Eqs. (1)-(4) by specifying that $\widetilde{\phi}=\widetilde{\phi}(x, y, z, t)$ indicates the electrostatic potential perturbation, $d \mathcal{W}_{s}=d v_{\|}\left(2 \pi B_{0} / m_{s}\right) d \mu_{0 s}$ is a volume element in velocity space and

$$
\Theta_{s}=\frac{T_{0_{\perp s}}}{T_{0_{\| s}}}, \quad \beta_{\perp_{s}}=8 \pi \frac{n_{0} T_{0_{\perp s}}}{B_{0}^{2}}
$$

are two parameters measuring, for each species $s$, the equilibrium temperature anisotropy and the ratio between perpendicular equilibrium kinetic pressure and magnetic pressure based on the guide field, respectively. The canonical bracket [, ], on the other hand, is defined as $[f, g]=\partial_{x} f \partial_{y} g-\partial_{y} f \partial_{x} g$ for two functions $f$ and $g$.

The model (1)-(4) is derived in Ref. [3] by performing an asymptotic expansion of the Boltzmann-Maxwell system, based on the following gyrokinetic ordering (adapted to our notation and assumptions):

$$
\begin{aligned}
& \frac{\omega}{\omega_{c s}} \sim \frac{\rho_{\perp_{s}}}{L} \sim \frac{k_{z}}{k_{\perp}} \sim \frac{U_{\perp}}{v_{A}} \sim \frac{\left|\widetilde{A}_{\|}\right|}{B_{0}} \sim \frac{U_{\|}}{v_{A}} \sim \frac{\widetilde{B}_{\|}}{B_{0}} \sim \frac{\widetilde{f}_{s}}{\mathcal{F}_{0 s}} \ll 1, \\
& k_{\perp} \rho_{\perp_{s}} \sim \Theta_{s} \sim \beta_{\perp_{s}} \sim \tau_{\perp_{s}} \sim 1 .
\end{aligned}
$$

In Eqs. (10)-(11) we introduced the following quantities:

$$
\rho_{\perp_{s}}=\frac{v_{\perp}}{\omega_{c s}}, \quad v_{A}=\frac{B_{0}}{\sqrt{4 \pi m_{i} n_{0}}}, \quad \tau_{\perp_{s}}=\frac{T_{0_{\perp s}}}{T_{0 \perp_{e}}}
$$

corresponding to the gyroradius of the particle of species $s$, to the Alfvén speed based on the guide field and to the ratio between the equilibrium perpendicular temperatures of the species $s$ and of the electron species, respectively. In Eqs. (10)-(11) we also denoted with $\omega, L, U_{\|}$and $U_{\perp}$, characteristic values of the frequency of the perturbations, of a macroscopic length scale of the system and of fluid velocities in the direction parallel to the guide field and in the plane perpendicular to it, respectively. In Eqs. (10)-(11), $k_{z}$, $k_{\perp}, \widetilde{A}_{\|}, \widetilde{B}_{\|}, \widetilde{f}_{s}$ and $\rho_{\perp_{s}}$ are to be intended as characteristic values of the corresponding quantities. 
Eqs. (10)-(11) determine also the regime of validity of the model. We remark that, apart from standard gyrokinetic assumptions such as low-frequency, strong anisotropy of the perturbations and finite gyroradius effects, the model is valid for values of $\beta_{\perp_{s}}$ of order unity, which is consistent with retaining the parallel magnetic perturbations $\widetilde{B}_{\|}$. Equilibrium temperature anistropy is also retained but strong anisotropies are not allowed $\left(\Theta_{s} \sim 1\right)$. Also, the perturbation of the gyrocenter distribution function is assumed to be a small disturbance of the equilibrium bi-Maxwellian. The latter actually also represents a particular choice for a spatially homogeneous distribution function which satisfies the Vlasov equation at the lowest order according to the ordering (10)(11) [3]. Our simple choice of the bi-Maxwellian allows for the inclusion of equilibrium temperature anisotropies and at the same time makes easier the comparison with previous reduced fluid or gyrofluid models (see, e.g. Ref. [21]) that could be derived assuming a Maxwellian distribution .

Further details about the properties of the model can be found in Ref. [3].

It is worth mentioning, at the end of this Section, that the $\delta f$ gyrokinetic model (1)-(4) can also be derived, in a way alternative to that described in Ref. [3], and which is based on well known gyrokinetic theory, the foundations thereof, are reviewed, for instance, in Ref. [1]. In particular, the $\delta f$ gyrokinetic equation (1) can be obtained from the gyrokinetic equation

$$
\frac{\partial f_{s}}{\partial t}+\dot{\mathbf{X}}_{s} \cdot \frac{\partial f_{s}}{\partial \mathbf{X}_{s}}+\dot{U}_{s} \frac{\partial f_{s}}{\partial U_{s}}=0
$$

where the dot indicate time derivatives along a gyrocenter orbit and $f_{s}=f_{s}\left(\mathbf{X}_{s}, U_{s}, \mu_{s}, t\right)$ is the full gyrocenter distribution function for the species $s$. The variables $\mathbf{X}_{s}, U_{s}$ and $\mu_{s}$ are gyrocenter variables obtained by means of near-identity transformations from the guiding center variables adopted for the unperturbed equilibrium state. These transformations are given explicitly, for instance, in Ref. [4]. In particular, the adiabatic invariant $\mu_{s}$ does not evolve in time (i.e. $\dot{\mu}_{s}=0$ ), whereas the evolution of $\mathbf{X}_{s}$ and $U_{s}$ 
is governed by [4]

$$
\begin{aligned}
& \dot{\mathbf{X}}_{s}=U_{s} \hat{z}+\frac{c}{B_{0}} \hat{z} \times \nabla\left(J_{0}\left(a_{s}\right) \widetilde{\phi}\left(\mathbf{X}_{s}, t\right)-\frac{U_{s}}{c} J_{0}\left(a_{s}\right) \widetilde{A}_{\|}\left(\mathbf{X}_{s}, t\right)+2 \frac{\mu_{0 s} B_{0}}{q_{s}} \frac{J_{1}\left(a_{s}\right)}{a_{s}} \frac{\widetilde{B}_{\|}}{B_{0}}\left(\mathbf{X}_{s}, t\right)\right), \\
& \dot{U}_{s}=-\frac{q_{s}}{m_{s} c} J_{0}\left(a_{s}\right) \frac{\partial \widetilde{A}_{\|}}{\partial t}\left(\mathbf{X}_{s}, t\right)-\frac{q_{s}}{m_{s}}\left(\frac { 1 } { B _ { 0 } } \left[J_{0}\left(a_{s}\right) \widetilde{A}_{\|}\left(\mathbf{X}_{s}, t\right), J_{0}\left(a_{s}\right) \widetilde{\phi}\left(\mathbf{X}_{s}, t\right)\right.\right. \\
& \left.\left.+2 \frac{\mu_{0 s} B_{0}}{q_{s}} \frac{J_{1}\left(a_{s}\right)}{a_{s}} \frac{\widetilde{B}_{\|}}{B_{0}}\left(\mathbf{X}_{s}, t\right)\right]+\frac{\partial}{\partial Z_{s}}\left(J_{0}\left(a_{s}\right) \widetilde{\phi}\left(\mathbf{X}_{s}, t\right)+2 \frac{\mu_{0 s} B_{0}}{q_{s}} \frac{J_{1}\left(a_{s}\right)}{a_{s}} \frac{\widetilde{B}_{\|}}{B_{0}}\left(\mathbf{X}_{s}, t\right)\right)\right)
\end{aligned}
$$

where spatial derivatives are taken with respect to the coordinates $\mathbf{X}_{s}$. Upon inserting into Eq. (13) the expressions for $\dot{\mathbf{X}}_{s}$ and $\dot{U}_{s}$ given by Eqs. (14) and (15) one obtains a gyrokinetic equation in a fixed Eulerian frame of coordinates. In the resulting equation one can insert the expression for the gyrocenter distribution function descending from the $\delta f$ approximation, i.e. $f_{s}=\mathcal{F}_{0 s}\left(U_{s}, \mu_{s}\right)+\widetilde{f}_{s}\left(\mathbf{X}_{s}, U_{s}, \mu_{s}, t\right)$. If one takes for the

function $\mathcal{F}_{0 s}$ the expression for the bi-Maxwellian given in Eq. (5) and applies the ordering (10)-(11), one obtains namely Eq. (1) as leading order evolution equation for $\tilde{f}_{s}$. We recall that, up to higher order terms negligible in this model, the expression for the adiabatic invariant $\mu_{s}$ is given by $[3,4]$

$$
\mu_{s}=\mu_{0 s}-2 \mu_{0 s} \frac{J_{1}\left(a_{s}\right)}{a_{s}} \frac{\widetilde{B}_{\|}}{B_{0}}\left(\mathbf{X}_{0 s}, t\right)+\frac{q_{s}}{B_{0}}\left(e^{i \mathbf{k} \cdot \boldsymbol{\rho}_{\perp s}}-J_{0}\left(a_{s}\right)\right)\left(\widetilde{\phi}\left(\mathbf{X}_{0 s}, t\right)-\frac{v_{\|}}{c} \widetilde{A}_{\|}\left(\mathbf{X}_{0 s}, t\right)\right)
$$

where $\boldsymbol{\rho}_{\perp s}=\left(1 / \omega_{c s}\right)(\hat{z} \times \mathbf{v})$, with $\mathbf{v}=v_{x} \hat{x}+v_{y} \hat{y}+v_{z} \hat{z}$, and $\mathbf{X}_{0 s}=\mathbf{x}-\boldsymbol{\rho}_{\perp s}$ is the vector indicating the guiding center position related to the species $s$.

\section{Hamiltonian formulation of the parent gyrokinetic model}

In this Section we present the Hamiltonian structure of the gyrokinetic model (1)-(4). Such structure is inherited from the Hamiltonian character of gyrocenter dynamics (see, e.g. Ref. [1]).

We recall that a dynamical system possesses a Hamiltonian structure (see, e.g. Refs. [22, 23]) if it can be cast in the following form:

$$
\frac{\partial \chi_{i}}{\partial t}=\left\{\chi_{i}, H\right\}, \quad i=1, \cdots, N
$$


In Eq. (17) $\chi_{1}, \cdots, \chi_{N}$ is a set of suitable dynamical variables (with $N$ positive integer) possibly depending on coordinates other than time. $H=H\left(\chi_{1}, \ldots, \chi_{N}\right)$ is the Hamiltonian functional (corresponding to the total energy of the system, conserved by the dynamics) and $\{$,$\} is a Poisson bracket, i.e. a bilinear antisymmetric operation$ satisfying the Leibniz and the Jacobi identity.

The Hamiltonian structure of the model (1)-(4) turns out to be analogous to that of some " $\delta f "$ drift-kinetic models $[24,25,26,20]$. In particular, physical intuition suggests that, in terms of the two dynamical variables $\chi_{1}=\widetilde{g}_{e}$ and $\chi_{2}=\widetilde{g}_{i}$, a candidate Hamiltonian functional is given by

$$
\begin{aligned}
& H\left(\widetilde{g}_{e}, \widetilde{g}_{i}\right)=\frac{1}{2} \sum_{s} \int d^{3} x d \mathcal{W}_{s}\left(T_{0_{\| s}} \frac{\widetilde{g}_{s}^{2}}{\mathcal{F}_{0 s}}\right. \\
& \left.+q_{s} \widetilde{g}_{s}\left(J_{0}\left(a_{s}\right) \widetilde{\phi}-\frac{v_{\|}}{c} J_{0}\left(a_{s}\right) \widetilde{A}_{\|}+2 \frac{\mu_{0 s} B_{0}}{q_{s}} \frac{J_{1}\left(a_{s}\right)}{a_{s}} \frac{\widetilde{B}_{\|}}{B_{0}}\right)\right),
\end{aligned}
$$

which can be shown, by direct computation, to be a quantity conserved by the dynamics. In terms of the actual gyrocenter perturbed distribution functions $\widetilde{f}_{s}$, the Hamiltonian (18) takes the form

$$
\begin{aligned}
& H\left(\widetilde{f}_{e}, \widetilde{f}_{i}\right)=\frac{1}{2} \sum_{s} \int d^{3} x d \mathcal{W}_{s}\left(T_{0_{\| s}} \frac{\widetilde{f}_{s}^{2}}{\mathcal{F}_{0 s}}\right. \\
& \left.+q_{s} \widetilde{f}_{s}\left(J_{0}\left(a_{s}\right) \widetilde{\phi}+\frac{v_{\|}}{c} J_{0}\left(a_{s}\right) \widetilde{A}_{\|}+2 \frac{\mu_{0 s} B_{0}}{q_{s}} \frac{J_{1}\left(a_{s}\right)}{a_{s}} \frac{\widetilde{B}_{\|}}{B_{0}}\right)\right),
\end{aligned}
$$

which is more perspicuous from the physical point of view. The first term on the right-hand side of Eq. (19) corresponds to the internal plasma free energy, whereas the three remaining contributions on the right-hand side, with the help of Eqs. (2)(4), yield the electromagnetic energy, with contributions coming from the polarization and magnetization effects. The candidate Hamiltonian functional (19) represents then the total energy of the system and generalizes the Hamiltonian functionals of Refs. $[24,25,26,20]$ by the inclusion of finite Larmor radius effects, parallel magnetic perturbations and equilibrium temperature anisotropy.

Analogy with Hamiltonian drift-kinetic models suggests that the Poisson bracket, 
expressed in terms of the variables $\widetilde{g}_{e}$ and $\widetilde{g}_{i}$, on the other hand, be given by

$$
\{F, G\}=-\sum_{s} \int d^{3} x d \mathcal{W}_{s}\left(\frac{c}{B_{0} q_{s}} \widetilde{g}_{s}\left[F_{\widetilde{g}_{s}}, G_{\widetilde{g}_{s}}\right]+\frac{v_{\|}}{T_{0_{\| s}}} \mathcal{F}_{0 s} F_{\widetilde{g}_{s}} \frac{\partial G_{\widetilde{g}_{s}}}{\partial z}\right) .
$$

The Poisson bracket (20) is the sum of two independent Poisson brackets, associated with the two particle species. The subscripts on the functionals $F$ and $G$ indicate functional derivatives so that, for instance, $F_{\widetilde{g}_{s}}=\delta F / \delta \widetilde{g}_{s}$.

Poisson brackets of the same form were presented in Refs. [24, 25, 26, 20], therefore we do not provide here a verification of the Poisson bracket properties, and in particular of the Jacobi identity.

We remark in particular that, the finite Larmor radius effects, as well as the parallel magnetic perturbations and temperature anisotropy effects present in this model, but not considered in Refs. [24, 25, 26, 20], intervene in the Hamiltonian, not in the Poisson bracket.

Direct computation shows that the Hamiltonian (18) and the Poisson bracket (20) indeed yield, using Eq. (17), the model equations (1). In order to see this it is convenient to note that, from Eq. (18) one has

$$
H_{\widetilde{g}_{s}}=T_{0_{\| s}} \frac{\widetilde{g}_{s}}{\mathcal{F}_{0 s}}+q_{s}\left(J_{0}\left(a_{s}\right) \widetilde{\phi}-\frac{v_{\|}}{c} J_{0}\left(a_{s}\right) \widetilde{A}_{\|}+\frac{2 \mu_{0 s} B_{0}}{q_{s}} \frac{J_{1}\left(a_{s}\right)}{a_{s}} \frac{\widetilde{B}_{\|}}{B_{0}}\right) .
$$

This relation follows from the property

$$
\begin{aligned}
& \int d^{3} x d \mathcal{W}_{s} f\left(\mathbf{x}, v_{\|}, \mu_{0 s}, t\right) \mathcal{J}_{i_{s s^{\prime}}} g\left(\mathbf{x}, v_{\|}, \mu_{s^{\prime}}, t\right) \\
& =\int d^{3} x d \mathcal{W}_{s^{\prime}} g\left(\mathbf{x}, v_{\|}, \mu_{s^{\prime}}, t\right) \mathcal{J}_{i_{s^{\prime}}} f\left(\mathbf{x}, v_{\|}, \mu_{0 s}, t\right),
\end{aligned}
$$

for two functions $f$ and $g$ and with $i=1,2,3, s=e, i$ and $s^{\prime}=e, i$. The operators $\mathcal{J}_{1_{s s^{\prime}}}, \mathcal{J}_{2_{s s^{\prime}}}, \mathcal{J}_{3_{s s^{\prime}}}$ are defined as

$$
\begin{aligned}
& \mathcal{J}_{1_{s s^{\prime}}} f=q_{s} J_{0}\left(a_{s}\right) L_{\phi_{s^{\prime}}} f, \quad \mathcal{J}_{2_{s s^{\prime}}} f=-q_{s} \frac{v_{\|}}{c} J_{0}\left(a_{s}\right) L_{A_{s^{\prime}}} f, \\
& \mathcal{J}_{3_{s s^{\prime}}} f=2 \mu_{0 s} \frac{J_{1}\left(a_{s}\right)}{a_{s}} L_{B_{s^{\prime}}} f
\end{aligned}
$$

for a function $f$. We indicated with $L_{\phi_{s}}, L_{A_{s}}$ and $L_{B_{s}}$ the operators allowing to express $\widetilde{\phi}, \widetilde{A}_{\|}$and $\widetilde{B}_{\|}$, respectively, in terms of $\widetilde{g}_{e}$ and $\widetilde{g}_{i}$ and which are defined in Eqs. (A.17), (A.24) and (A.18), respectively. 


\section{Hamiltonian reduction to gyrofluid models}

In this Section, we carry out a reduction of the parent model (1)-(4) leading to gyrofluid models evolving an arbitrary number of moments. The reduction is such to preserve the existence of a Hamiltonian structure in the resulting gyrofluid models and can be carried out in two steps. A first Hamiltonian reduction, described in Sec. 4.1, leads from the parent model (1)-(4) to a Hamiltonian model evolving the perturbation of the gyrocenter distribution function averaged with respect to the magnetic moment. The second step leads from the latter model to the Hamiltonian gyrofluid models.

4.1. First Hamiltonian reduction: from gyrocenter distribution functions to averaged distribution functions

We begin the procedure by assuming that the following decomposition is valid:

$$
\widetilde{g}_{s}\left(\mathbf{x}, v_{\|}, \mu_{0 s}, t\right)=\mathrm{f}_{e q_{s}}\left(\mu_{0 s}\right) \sum_{n=0}^{+\infty} L_{n}\left(\frac{\mu_{0 s} B_{0}}{T_{0_{\perp s}}}\right) g_{n_{s}}\left(\mathbf{x}, v_{\|}, t\right),
$$

where the $L_{n}$, with non-negative integer $n$, are the Laguerre polynomials and

$$
\mathrm{f}_{e q_{s}}\left(\mu_{0 s}\right)=\frac{m_{s}}{2 \pi T_{0_{\perp s}}} \mathrm{e}^{-\frac{\mu_{0 s} B_{0}}{T_{0 \perp s}}}
$$

Due to the orthogonality of Laguerre polynomials, we have the relations

$$
g_{l_{s}}\left(\mathbf{x}, v_{\|}, t\right)=\frac{2 \pi B_{0}}{m_{s}} \int d \mu_{0 s} L_{l}\left(\frac{\mu_{0 s} B_{0}}{T_{0_{\perp s}}}\right) \widetilde{g}_{s}\left(\mathbf{x}, v_{\|}, \mu_{0 s}, t\right)
$$

for any non-negative integer $l$. The functions $g_{l_{s}}$ correspond, for each species $s$, to moments of the generalized distribution function $\widetilde{g}_{s}$, with respect to Laguerre polynomials in the normalized squared perpendicular velocity $\mu_{0 s} B_{0} / T_{0_{\perp s}}=v_{\perp}^{2} / v_{t h \perp s}^{2}$, where $v_{t h_{\perp s}}=\left(T_{0_{\perp s}} / m_{s}\right)^{1 / 2}$ is the thermal perpendicular velocity associated with the species $s$.

We can also transfer the decomposition (24) to the gyrocenter distribution function $\widetilde{f}_{s}$ in the following way:

$$
\widetilde{f}_{s}\left(\mathbf{x}, v_{\|}, \mu_{0 s}, t\right)=\mathrm{f}_{e q_{s}}\left(\mu_{0 s}\right) \sum_{n=0}^{+\infty} L_{n}\left(\frac{\mu_{0 s} B_{0}}{T_{0_{\perp s}}}\right) f_{n_{s}}\left(\mathbf{x}, v_{\|}, t\right) .
$$


Hamiltonian gyrofluid reductions of gyrokinetic equations

Equation (24) can then be written as

$$
\begin{aligned}
& \widetilde{g}_{s}\left(\mathbf{x}, v_{\|}, \mu_{0 s}, t\right)=\mathrm{f}_{e q_{s}}\left(\mu_{0 s}\right)\left(\sum_{n=0}^{+\infty} L_{n}\left(\frac{\mu_{0 s} B_{0}}{T_{0_{\perp s}}}\right) f_{n_{s}}\left(\mathbf{x}, v_{\|}, t\right)+\frac{q_{s}}{T_{0_{\| s}}} \frac{v_{\|}}{c} F_{e q_{s}}\left(v_{\|}\right) J_{0}\left(a_{s}\right) \widetilde{A}_{\|}(\mathbf{x}, t)\right) \\
& =\mathrm{f}_{e q_{s}}\left(\mu_{0 s}\right) \sum_{n=0}^{+\infty} L_{n}\left(\frac{\mu_{0 s} B_{0}}{T_{0_{\perp s}}}\right)\left(f_{n_{s}}\left(\mathbf{x}, v_{\|}, t\right)+\frac{q_{s}}{T_{0_{\| s}}} \frac{v_{\|}}{c} F_{e q_{s}}\left(v_{\|}\right) \frac{\mathrm{e}^{-\frac{k_{\perp}^{2} \rho_{t h_{\perp s}}^{2}}{2}}}{n !}\left(\frac{k_{\perp}^{2} \rho_{t h_{\perp s}}^{2}}{2}\right)^{n} \widetilde{A}_{\|}(\mathbf{x}, t)\right),
\end{aligned}
$$

where

$$
F_{e q_{s}}\left(v_{\|}\right)=n_{0} \sqrt{\frac{m_{s}}{2 \pi T_{0_{\| s}}}} \mathrm{e}^{-\frac{m_{s} v_{\|}^{2}}{2 T_{0} \| s}} .
$$

Note that $F_{e q_{s}}\left(v_{\|}\right) \mathbf{f}_{e q_{s}}\left(\mu_{0 s}\right)=\mathcal{F}_{0 s}\left(v_{\|}, \mu_{0 s}\right)$. We also remark that, for the last step of Eq. (28), we made use of the relation [27]

$$
J_{0}\left(a_{s}\right)=J_{0}\left(k_{\perp} \rho_{t h_{\perp s}} \sqrt{\frac{2 \mu_{0 s} B_{0}}{T_{0_{\perp s}}}}\right)=\mathrm{e}^{-\frac{k_{\perp}^{2} \rho_{t h}^{2}}{2}} \sum_{n=0}^{+\infty} \frac{1}{n !} L_{n}\left(\frac{\mu_{0 s} B_{0}}{T_{0_{\perp s}}}\right)\left(\frac{k_{\perp}^{2} \rho_{t h_{\perp s}}^{2}}{2}\right)^{n}
$$

with $\rho_{t h_{\perp s}}=\sqrt{T_{0_{\perp s}} / m_{s}} / \omega_{c s}$ indicating the perpendicular thermal radius for the species $s$.

Upon introducing the gyroverage operators

$$
\begin{aligned}
& \mathcal{G}_{1 n_{s}}=\frac{2 \pi B_{0}}{m_{s}} \int d \mu_{0 s} \mathrm{f}_{e q_{s}}\left(\mu_{0 s}\right) L_{n}\left(\frac{\mu_{0 s} B_{0}}{T_{0_{\perp s}}}\right) J_{0}\left(a_{s}\right) \\
& \mathcal{G}_{2 n_{s}}=\frac{2 \pi B_{0}}{m_{s}} \int d \mu_{0 s} \mathrm{f}_{e q_{s}}\left(\mu_{0 s}\right) L_{n}\left(\frac{\mu_{0 s} B_{0}}{T_{0_{\perp s}}}\right) \frac{\mu_{0 s} B_{0}}{T_{0_{\perp s}}} \frac{J_{1}\left(a_{s}\right)}{a_{s}}, \quad n=0,1,2, \cdots
\end{aligned}
$$

from Eqs. (26) and (28) it also follows that

$$
g_{l_{s}}=f_{l_{s}}+\frac{q_{s}}{T_{0_{\| s}}} \frac{v_{\|}}{c} F_{e q_{s}}\left(v_{\|}\right) \mathcal{G}_{1 l_{s}} \widetilde{A}_{\|}
$$

Applying the expansion (24), the Hamiltonian (18) can be transformed in terms of the new variables $g_{l_{s}}$ as

$$
\begin{aligned}
& H\left(g_{0_{e}}, g_{1_{e}}, \cdots, g_{0_{i}}, g_{1_{i}}, \cdots\right)=\frac{1}{2} \sum_{s} \sum_{n=0}^{+\infty} \int d^{3} x d v_{\|}\left(T_{0_{\| s}} \frac{g_{n_{s}}^{2}}{F_{e q_{s}}}\right. \\
& \left.+q_{s} g_{n_{s}}\left(\mathcal{G}_{1 n_{s}} \widetilde{\phi}-\frac{v_{\|}}{c} \mathcal{G}_{1 n_{s}} \widetilde{A}_{\|}+2 \frac{T_{0_{\perp s}}}{q_{s}} \mathcal{G}_{2 n_{s}} \frac{\widetilde{B}_{\|}}{B_{0}}\right)\right) .
\end{aligned}
$$


Hamiltonian gyrofluid reductions of gyrokinetic equations

As far as the Poisson bracket (20) is concerned, we first remark that, by the chain rule for functional derivatives, one has the relation

$$
F_{\widetilde{g}_{s}}=\sum_{n=0}^{+\infty} L_{n}\left(\frac{\mu_{0 s} B_{0}}{T_{0_{\perp s}}}\right) \bar{F}_{g_{n_{s}}}
$$

for functionals $F$ and $\bar{F}$ such that $F\left(\widetilde{g}_{s}\right)=\bar{F}\left(g_{0_{s}}, g_{1_{s}}, \cdots\right)$.

With the help of the relation (35), the Poisson bracket (20) can be expressed in terms of the new variables in the following way:

$$
\begin{aligned}
& \{F, G\}=-\sum_{s} \int d^{3} x d v_{\|}\left(\frac{c}{B_{0} q_{s}} \sum_{n, n^{\prime}=0}^{+\infty} \sum_{p=\left|n^{\prime}-n\right|}^{n^{\prime}+n} C_{n n^{\prime} p} g_{p_{s}}\left[F_{g_{n_{s}}}, G_{g_{n^{\prime} s}}\right]\right. \\
& \left.+\frac{v_{\|}}{T_{0_{\| s}}} F_{e q_{s}} \sum_{n=0}^{+\infty} F_{g_{n_{s}}} \frac{\partial}{\partial z} G_{g_{n_{s}}}\right)
\end{aligned}
$$

where the constants $C_{n n^{\prime} p}$ permit to express a product of Laguerre polynomials as a linear combination of such polynomials in the following way:

$$
L_{n}(x) L_{n^{\prime}}(x)=\sum_{p=\left|n^{\prime}-n\right|}^{n^{\prime}+n} C_{n n^{\prime} p} L_{p}(x)
$$

The expressions for the constants $C_{n n^{\prime} p}$ are derived, for instance, in Ref. [28], and are given by

$$
C_{n n^{\prime} p}=\left(-\frac{1}{2}\right)^{n+n^{\prime}-p} \sum_{m} \frac{\left(n+n^{\prime}-m\right) !}{(n-m) !\left(n^{\prime}-m\right) !\left(2 m-n-n^{\prime}+p\right) !\left(n+n^{\prime}-p-m\right) !}
$$

where the sum over the integers $m$ is defined by requiring that none of the arguments of the factorials be negative.

We perform a reduction on the expansion (24) by imposing that

$$
f_{l_{s}}=0, \quad \text { for } l \geq 1, \quad s=e, i
$$

In terms of the generalized distribution functions $g_{l_{s}}$, the reduction (39) corresponds to

$$
g_{l_{s}}=\frac{q_{s}}{T_{0_{\| s}}} \frac{v_{\|}}{c} F_{e q_{s}}\left(v_{\|}\right) \mathcal{G}_{1 l_{s}} \widetilde{A}_{\|}, \quad \text { for } l \geq 1, \quad s=e, i
$$

The condition (39) amounts to setting to zero all moments of the gyrocenter distribution function, involving finite powers of the perpendicular velocity. For instance, 
this amounts to setting equal to zero the gyrofluid perpendicular temperature and perpendicular heat flux fluctuations. Although this is a rather strong restriction, it could be suitable, for instance, to plasmas for which the evolution of the perpendicular temperature fluctuations (in terms of particle moments) is adiabatic.

As a consequence of the imposed conditions (40), the Hamiltonian (34) becomes

$$
\begin{aligned}
& H\left(g_{0_{e}}, g_{0_{i}}\right)=\frac{1}{2} \sum_{s} \int d^{3} x d v_{\|}\left(T_{0_{\| s}} \frac{g_{0_{s}}{ }^{2}}{F_{e q_{s}}}\right. \\
& \left.+q_{s} g_{0_{s}}\left(\mathcal{G}_{10_{s}} \widetilde{\phi}-\frac{v_{\|}}{c} \mathcal{G}_{10_{s}} \widetilde{A}_{\|}+2 \frac{T_{0_{\perp s}}}{q_{s}} \mathcal{G}_{20_{s}} \frac{\widetilde{B}_{\|}}{B_{0}}\right)\right) .
\end{aligned}
$$

On the other hand, the relations (2), (3) and (4), after the truncation, read

$$
\begin{aligned}
& \sum_{s} q_{s} \mathcal{G}_{10_{s}} \int d v_{\|} g_{0_{s}}=n_{0} \sum_{s}\left(\frac{q_{s}^{2}}{T_{0_{\perp s}}}\left(1-\Gamma_{0}\left(b_{s}\right)\right) \widetilde{\phi}-q_{s}\left(\Gamma_{0}\left(b_{s}\right)-\Gamma_{1}\left(b_{s}\right)\right) \frac{\widetilde{B}_{\|}}{B_{0}}\right), \\
& \sum_{s}\left(q_{s} \mathcal{G}_{10_{s}} \int d v_{\|} v_{\|} g_{0_{s}}-\frac{q_{s}^{2} n_{0}}{m_{s} c} \mathcal{G}_{10_{s}}^{2} \widetilde{A}_{\|}\right) \\
& =-\frac{c}{4 \pi} \nabla_{\perp}^{2} \widetilde{A}_{\|}+\sum_{s} \frac{q_{s}^{2} n_{0}}{m_{s} c}\left(1-\frac{1}{\Theta_{s}}\right)\left(1-\Gamma_{0}\left(b_{s}\right)\right) \widetilde{A}_{\|}, \\
& \sum_{s} 2 \frac{\beta_{\perp_{s}}}{n_{0}} \mathcal{G}_{20_{s}} \int d v_{\|} g_{0_{s}}=-\sum_{s} \beta_{\perp_{s}} \frac{q_{s}}{T_{0_{\perp s}}}\left(\Gamma_{0}\left(b_{s}\right)-\Gamma_{1}\left(b_{s}\right)\right) \widetilde{\phi} \\
& -\left(2+2 \sum_{s} \beta_{\perp_{s}}\left(\Gamma_{0}\left(b_{s}\right)-\Gamma_{1}\left(b_{s}\right)\right)\right) \frac{\widetilde{B}_{\|}}{B_{0}}
\end{aligned}
$$

where $\Gamma_{n}\left(b_{s}\right)=I_{n}\left(b_{s}\right) \exp \left(-b_{s}\right)$, with $I_{n}$ indicating the modified Bessel function of order $n$ and $b_{s}=k_{\perp}^{2} \rho_{t h_{\perp s}}^{2}$. In order to derive Eq. (43), use was made of the relation $\sum_{n=0}^{+\infty} \mathcal{G}_{1 n_{s}}^{2}=\Gamma_{0}\left(b_{s}\right)$. Following the arguments provided in the Appendix, it is possible to find conditions for expressing $\widetilde{\phi}, \widetilde{A}_{\|}$and $\widetilde{B}_{\|}$in terms of the $g_{0_{s}}$. With the electromagnetic fields expressed in terms of the generalized distribution functions $g_{0_{s}}$, one sees that the Hamiltonian (41) effectively only depends on $g_{0_{e}}$ and $g_{0_{i}}$. For functionals $F$ and $G$ which, as the Hamiltonian (41), only depend on $g_{0_{s}}$, the Poisson bracket (36) reduces to

$$
\{F, G\}=-\sum_{s} \int d^{3} x d v_{\|}\left(\frac{c}{B_{0} q_{s}} g_{0_{s}}\left[F_{g_{0_{s}}}, G_{g_{0_{s}}}\right]-\frac{v_{\|}}{T_{0_{\| s}}} F_{e q_{s}} F_{g_{0_{s}}} \frac{\partial}{\partial z} G_{g_{0_{s}}}\right) .
$$

Because $\{F, G\}$ is also a functional of the $g_{0_{s}}$ only, the functionals of $g_{0_{i}}$ and $g_{0_{e}}$ form a sub-algebra with respect to the Poisson bracket (36). This guarantees that the reduced 
system preserves a Hamiltonian structure (in particular, the bracket (45) satisfies the properties of a Poisson bracket), and the evolution of the observables of the system is governed by the Hamiltonian (41) and the Poisson bracket (45). Following Eq. (17), the evolution of the averaged distribution functions $g_{0_{s}}$, for a species $s$, is given by

$$
\begin{aligned}
& \frac{\partial g_{0_{s}}}{\partial t}+\frac{c}{B_{0}}\left[\mathcal{G}_{10_{s}} \widetilde{\phi}-\frac{v_{\|}}{c} \mathcal{G}_{10_{s}} \widetilde{A}_{\|}+2 \frac{T_{0_{\perp s}}}{q_{s}} \mathcal{G}_{20_{s}} \frac{\widetilde{B}_{\|}}{B_{0}}, g_{0_{s}}\right] \\
& +v_{\|} \frac{\partial}{\partial z}\left(g_{0_{s}}+\frac{q_{s}}{T_{0_{\| s}}} F_{e q_{s}}\left(\mathcal{G}_{10_{s}} \widetilde{\phi}-\frac{v_{\|}}{c} \mathcal{G}_{10_{s}} \widetilde{A}_{\|}+2 \frac{T_{0_{\perp s}}}{q_{s}} \mathcal{G}_{20_{s}} \frac{\widetilde{B}_{\|}}{B_{0}}\right)\right)=0 .
\end{aligned}
$$

Eq. (46), complemented by Eqs. (42)-(44), provides a system that governs the evolution of the gyrocenter distribution functions averaged over the magnetic moment (recall that, from Eqs. (6) and (26), we have $\left.g_{0_{s}}=\left(2 \pi B_{0} / m_{s}\right) \int d \mu_{0 s}\left(\widetilde{f}_{s}+\left(q_{s} / T_{0_{\| s}}\right)\left(v_{\|} / c\right) \mathcal{F}_{0 s} J_{0} \widetilde{A}_{\|}\right)\right)$ in such a way that the Hamiltonian character of the parent (not averaged) gyrokinetic model is preserved. This generalizes previous Hamiltonian models for drift-kinetic equations [24, 20], by taking into account parallel magnetic fluctuations, finite Larmor radius effects and equilibrium temperature anisotropies. From it, one can also obtain, in the appropriate collisionless limit, the hybrid fluid-kinetic model of Ref. [29].

\subsection{Second Hamiltonian reduction: from averaged distribution functions to gyrofluid moments}

In this second stage, we consider as parent model the Hamiltonian model derived in Sec. 4.1 and consisting of Eqs. (46), (42)-(44). It turns out that, in order to obtain, from such model, a Hamiltonian gyrofluid model, it is possible to extend the procedure adopted in Ref. [20], which we will refer to in the following. As a result, a class of Hamiltonian gyrofluid models for moments of arbitrary order in the parallel velocity coordinate, will be derived.

We begin by assuming that the following decomposition of the functions $g_{0_{s}}$ in 
terms of Hermite polynomials is valid:

$$
\begin{aligned}
& g_{0_{s}}\left(\mathbf{x}, v_{\|}, t\right)=\sum_{n=0}^{+\infty} \frac{g_{0 n_{s}}(\mathbf{x}, t)}{\sqrt{n !}} H_{n}\left(\frac{v_{\|}}{v_{t h_{\| s}}}\right) F_{e q_{s}}\left(v_{\|}\right), \\
& =\sum_{n=0}^{+\infty} \frac{f_{0 n_{s}}(\mathbf{x}, t)}{\sqrt{n !}} H_{n}\left(\frac{v_{\|}}{v_{t h_{\| s}}}\right) F_{e q_{s}}\left(v_{\|}\right)+\frac{q_{s}}{T_{0_{\| s}}} \frac{v_{\|}}{c} F_{e q_{s}}\left(v_{\|}\right) \mathcal{G}_{10_{s}} \widetilde{A}_{\|}(\mathbf{x}, t) .
\end{aligned}
$$

In Eq. (47), $H_{n}$ indicates the Hermite polynomial of order $n$, with $n$ a non-negative integer. The functions $g_{0 n_{s}}$ and $f_{0 n_{s}}$ are then proportional to moments, with respect to the Hermite polynomials in the normalized parallel velocity coordinate $v_{\|} / v_{t h_{\| s}}$, of the averaged distribution functions $g_{0_{s}}$ and $f_{0_{s}}$, respectively. Indeed, due to the orthogonality relation of Hermite polynomials, one has

$$
g_{0 n_{s}}=\frac{1}{n_{0} \sqrt{n !}} \int d v_{\|} H_{n} g_{0_{s}}, \quad f_{0 n_{s}}=\frac{1}{n_{0} \sqrt{n !}} \int d v_{\|} H_{n} f_{0_{s}} .
$$

From the definition (47), it follows that $g_{0 n_{s}}=f_{0 n_{s}}$ for $n \neq 1$, and $g_{01_{s}}=f_{01_{s}}+$ $\left(q_{s} / T_{0_{\| s}}\right)\left(v_{t h_{\| s}} / c\right) \mathcal{G}_{10_{s}} \widetilde{A}_{\|}$, where we also made use of the fact that $H_{1}\left(v_{\|} / v_{t h_{\| s}}\right)=v_{\|} / v_{t h_{\| s}}$. In order to exemplify the relation between the moments $g_{0 n_{s}}$ and the usually adopted gyrofluid moments, we specify that the following relations hold:

$$
\begin{gathered}
g_{00_{s}}=\frac{\widetilde{N}_{s}}{n_{0}}, \quad g_{01_{s}}=\frac{\widetilde{U}_{s}}{v_{t h_{\| s}}}+\frac{q_{s}}{m_{s} v_{t h_{\| s}} c} \mathcal{G}_{10_{s}} \widetilde{A}_{\|}, \\
g_{02_{s}}=\frac{\widetilde{T}_{\|_{s}}}{\sqrt{2} m_{s} v_{t h_{\| s}}^{2}}, \quad g_{03_{s}}=\sqrt{\frac{2}{3} \frac{\widetilde{Q}_{\|_{s}}}{n_{0} T_{0_{\| s} v_{t h_{\| s}}}}}
\end{gathered}
$$

where $\widetilde{N}_{s}=\int d v_{\|} f_{0_{s}}, \widetilde{U}_{s}=\int d v_{\|} v_{\|} f_{0_{s}} / n_{0}, \widetilde{T}_{\|_{s}}=\left(m_{s} v_{t h_{\| s}}^{2} / n_{0}\right) \int d v_{\|}\left(v_{\|}^{2} / v_{t h_{\| s}}^{2}-\right.$ 1) $f_{0_{s}}, \widetilde{Q}_{\|_{s}}=\left(m_{s} v_{t h_{\| s}}^{3} / 2\right) \int d v_{\|}\left(v_{\|}^{3} / v_{t h_{\| s}}^{3}-3 v_{\|} / v_{t h_{\| s}}\right) f_{0_{s}}$ are the fluctuations of the gyrofluid density, parallel velocity, parallel temperature and parallel heat flux, respectively, for the species $s$.

From the relation (48) and from Eq. (46), one can obtain the following hierarchy 
of evolution equations for the functions $g_{0 n_{s}}$ :

$$
\begin{aligned}
& \frac{\partial g_{0 n_{s}}}{\partial t}+\frac{c}{B_{0}}\left[\mathcal{G}_{10_{s}} \widetilde{\phi}+\frac{2 T_{0_{\perp s}}}{q_{s}} \mathcal{G}_{20_{s}} \frac{\widetilde{B}_{\|}}{B_{0}}, g_{0 n_{s}}\right]-\sqrt{n+1} \frac{v_{t h_{\| s}}}{B_{0}}\left[\mathcal{G}_{10_{s}} \widetilde{A}_{\|}, g_{0 n+1_{s}}\right]-\sqrt{n} \frac{v_{t h_{\| s}}}{B_{0}}\left[\mathcal{G}_{10_{s}} \widetilde{A}_{\|}, g_{0 n-1_{s}}\right] \\
& +\sqrt{n+1} v_{t h_{\| s}} \frac{\partial g_{0 n+1_{s}}}{\partial z}+\sqrt{n} v_{t h_{\| s}} \frac{\partial g_{0 n-1_{s}}}{\partial z}+\delta_{n 1} \frac{q_{s}}{T_{0_{\| s}}} v_{t h_{\| s}} \frac{\partial}{\partial z}\left(\mathcal{G}_{10_{s}} \widetilde{\phi}+\frac{2 T_{0_{\perp s}}}{q_{s}} \mathcal{G}_{20_{s}} \frac{\widetilde{B}_{\|}}{B_{0}}\right) \\
& -\left(\delta_{n 0}+\delta_{n 2}\right) \sqrt{n !} \frac{q_{s}}{T_{0_{\| s}}} \frac{v_{t h_{\| s}}^{2}}{c} \frac{\partial}{\partial z} \mathcal{G}_{10_{s}} \widetilde{A}_{\|}=0, \quad n=0,1,2, \cdots
\end{aligned}
$$

where use was made of the relation $x H_{n}(x)=H_{n+1}(x)+n H_{n-1}(x)$. These equations are complemented by the relations (42)-(44) for the electromagnetic fields which, in terms of the moments $g_{0 n_{s}}$, become

$$
\begin{aligned}
& \sum_{s} q_{s} \mathcal{G}_{10_{s}} g_{00_{s}}=\sum_{s}\left(\frac{q_{s}^{2}}{T_{0_{\perp s}}}\left(1-\Gamma_{0}\left(b_{s}\right)\right) \widetilde{\phi}-q_{s}\left(\Gamma_{0}\left(b_{s}\right)-\Gamma_{1}\left(b_{s}\right)\right) \frac{\widetilde{B}_{\|}}{B_{0}}\right) \\
& \sum_{s} \mathcal{G}_{10_{s}}\left(n_{0} q_{s} v_{t h_{\| s}} g_{01_{s}}-\frac{q_{s}^{2} n_{0}}{m_{s} c} \mathcal{G}_{10_{s}} \widetilde{A}_{\|}\right) \\
& =-\frac{c}{4 \pi} \nabla_{\perp}^{2} \widetilde{A}_{\|}+\sum_{s} \frac{q_{s}^{2} n_{0}}{m_{s} c}\left(1-\frac{1}{\Theta_{s}}\right)\left(1-\Gamma_{0}\left(b_{s}\right)\right) \widetilde{A}_{\|} \\
& \sum_{s} 2 \beta_{\perp_{s}} \mathcal{G}_{20_{s}} g_{00_{s}}=-\sum_{s} \beta_{\perp_{s}} \frac{q_{s}}{T_{0_{\perp s}}}\left(\Gamma_{0}\left(b_{s}\right)-\Gamma_{1}\left(b_{s}\right)\right) \widetilde{\phi} \\
& -\left(2+2 \sum_{s} \beta_{\perp_{s}}\left(\Gamma_{0}\left(b_{s}\right)-\Gamma_{1}\left(b_{s}\right)\right)\right) \frac{\widetilde{B}_{\|}}{B_{0}}
\end{aligned}
$$

From Eq. (51) one can see that the evolution equation of $g_{0 n_{s}}$, for a generic $n$, depends on the higher order moment $g_{0 n+1_{s}}$. The hierarchy is thus not closed and some additional constraints are required if one intends to reduce the infinite system, given by Eqs. (51), to a closed system with a finite number of equations. However, we can first remark that, by a simple extension of the arguments provided in the Appendix, when the relations (52)-(54) can be inverted, one can write

$$
\widetilde{\phi}=\sum_{s} \mathcal{L}_{\phi_{s}} g_{00_{s}}, \quad \widetilde{A}_{\|}=\sum_{s} \mathcal{L}_{A_{s}} g_{01_{s}}, \quad \widetilde{B}_{\|}=\sum_{s} \mathcal{L}_{B_{s}} g_{00_{s}}
$$

for appropriate linear symmetric operators $\mathcal{L}_{\phi_{s}}, \mathcal{L}_{A_{s}}$ and $\mathcal{L}_{B_{s}}$. When this property is taken into account, one sees that the system (51) falls into the framework treated in Ref. [20] (more precisely, Eq. (51) has to be compared with Eq. (26) of Ref. [20]). 
In such reference, it was shown that infinite hierarchy of the type of Eq. (51) can be truncated at an arbitrary order, while preserving the Hamiltonian structure. This is accomplished by imposing

$$
g_{0 \mathcal{N}_{s}+1_{s}}=\alpha_{s} g_{0 \mathcal{N}_{s s}}, \quad s=e, i
$$

where $\mathcal{N}_{e, i}$ are arbitrary positive integers that fix the desired order of truncation for each species, and $\alpha_{e, i}$ are real constants.

The truncated system of evolution equations resulting from imposing the closures (56) to the hierarchy (51), can then be written as

$$
\begin{aligned}
& \frac{\partial g_{0 m_{s}}}{\partial t}+\frac{c}{B_{0}}\left[\mathcal{G}_{10_{s}} \widetilde{\phi}+\frac{2 T_{0_{\perp s}}}{q_{s}} \mathcal{G}_{20_{s}} \frac{\widetilde{B}_{\|}}{B_{0}}, g_{0 m_{s}}\right]-\frac{v_{t h_{\| s}}}{B_{0}}\left[\mathcal{G}_{10_{s}} \widetilde{A}_{\|}, W_{s_{m n}} g_{0 n_{s}}\right] \\
& +v_{t h_{\| s}} \frac{\partial}{\partial z} W_{s_{m n}} g_{0 n_{s}}+\delta_{m 1} v_{t h_{\| s}} \frac{q_{s}}{T_{0_{\| s}}} \frac{\partial}{\partial z}\left(\mathcal{G}_{10_{s}} \widetilde{\phi}+\frac{2 T_{0_{\perp s}}}{q_{s}} \mathcal{G}_{20_{s}} \frac{\widetilde{B}_{\|}}{B_{0}}\right) \\
& -\sqrt{m !}\left(\delta_{m 0}+\delta_{m 2}\right) v_{t h_{\| s}}^{2} \frac{q_{s}}{T_{0_{\| s}} c} \frac{\partial}{\partial z} \mathcal{G}_{10_{s}} \widetilde{A}_{\|}=0, \quad 0 \leq m \leq \mathcal{N}_{s}
\end{aligned}
$$

where the sum over the repeated index $n$ is understood and $W_{s_{m n}}$ indicate the elements of a pair of symmetric matrices $W_{s}$, for $s=e, i$, defined as

$$
W_{s_{m n}}=\sqrt{m} \delta_{m, n+1}+\sqrt{m+1} \delta_{m, n-1}+\alpha_{s} \sqrt{\mathcal{N}_{s}+1} \delta_{m, \mathcal{N}_{s}} \delta_{n, \mathcal{N}_{s}}, \quad
$$

for $0 \leq m \leq \mathcal{N}_{s}, 0 \leq n \leq \mathcal{N}_{s}$ and $s=e, i$. We establish as a convention in this paper, that in the expression $A_{m n}$ for the element of a matrix $A$, the first index $m$ refers to the row of the matrix, and the second index $n$ to the column.

As described in Ref. [20], it is convenient, for a system of the type (57), to introduce, for each species, the alternative set of dynamical variables $G_{i_{s}}$ defined as

$$
G_{i_{s}}(\mathbf{x}, t)=\mathcal{U}_{s_{i m}}^{T} g_{0 m_{s}}(\mathbf{x}, t), \quad 0 \leq i_{s} \leq \mathcal{N}_{s}, \quad s=e, i
$$

In Eq. (59) $\mathcal{U}_{s_{i m}}$ indicate the elements of the pair of orthogonal matrices $\mathcal{U}_{s}$ such that, for each species $s, \mathcal{U}_{s}^{T} W_{s} \mathcal{U}_{s}=\Lambda_{s}$, where $\Lambda_{s}=\operatorname{diag}\left(\lambda_{0_{s}}, \lambda_{1_{s}}, \cdots, \lambda_{\mathcal{N}_{s}}\right)$ with $\lambda_{0_{s}}, \lambda_{1_{s}}, \cdots, \lambda_{\mathcal{N}_{s}}$ the eigenvalues of the matrix $W_{s}$. In terms of such variables, in fact, the Poisson bracket 
for the truncated Hamiltonian system (57) (complemented by the relations (55)) takes the remarkably simpler form [20]

$$
\{F, G\}=-\sum_{s} \sum_{i=0}^{\mathcal{N}_{s}}\left(\frac{c}{q_{s} B_{0} n_{0} v_{\left(i_{s}\right)}} \int d^{3} x G_{i_{s}}\left[\frac{\delta F}{\delta G_{i_{s}}}, \frac{\delta G}{\delta G_{i_{s}}}\right]+\frac{v_{t h_{\| s}}}{T_{0_{\| s} n_{0}}} \lambda_{i_{s}} \int d^{3} x \frac{\delta F}{\delta G_{i_{s}}} \frac{\partial}{\partial z} \frac{\delta G}{\delta G_{i_{s}}}\right),
$$

where the constants $v_{\left(i_{s}\right)}$ are determined by imposing that the eigenvectors of the matrices $\mathcal{U}_{s}$ be orthonormal. In particular we notice that, for each species $s$, the matrices $\mathcal{U}_{s}$ have the form

$$
\mathcal{U}_{s}=\left(\begin{array}{ccccc}
v_{\left(0_{s}\right)} & v_{\left(1_{s}\right)} & \ldots & \ldots & v_{\left(\mathcal{N}_{s}\right)} \\
\lambda_{0_{s}} v_{\left(0_{s}\right)} & \lambda_{1_{s}} v_{\left(1_{s}\right)} & \ldots & \ldots & \lambda_{\mathcal{N}_{s}} v_{\left(\mathcal{N}_{s}\right)} \\
\frac{\lambda_{0_{s}}^{2}-1}{\sqrt{2}} v_{\left(0_{s}\right)} & \frac{\lambda_{1_{s}}^{2}-1}{\sqrt{2}} v_{\left(1_{s}\right)} & \ldots & \ldots & \frac{\lambda_{\mathcal{N}_{s}}^{2}-1}{\sqrt{2}} v_{\left(\mathcal{N}_{s}\right)} \\
\cdot & \cdot & \ldots & \ldots & . \\
\cdot & \cdot & \ldots & \ldots & .
\end{array}\right),
$$

We notice also that, in terms of the variables $G_{i_{s}}$, Eqs. (57) take the simpler form

$$
\begin{aligned}
& \frac{\partial G_{i_{s}}}{\partial t}+\frac{c}{B_{0}}\left[\mathcal{G}_{10_{s}} \widetilde{\phi}+\frac{2 T_{0_{\perp s}}}{q_{s}} \mathcal{G}_{20_{s}} \frac{\widetilde{B}_{\|}}{B_{0}}-\lambda_{i_{s}} \frac{v_{t h_{\| s}}}{c} \mathcal{G}_{10_{s}} \widetilde{A}_{\|}, G_{i_{s}}\right]+v_{t h_{\| s}} \lambda_{i_{s}} \frac{\partial G_{i_{s}}}{\partial z} \\
& +v_{t h_{\| s}} \sqrt{m !} U_{s_{i m}}^{T}\left(\delta_{m 1} \frac{q_{s}}{T_{0_{\| s}}} \frac{\partial}{\partial z}\left(\mathcal{G}_{10_{s}} \widetilde{\phi}+\frac{2 T_{0_{\perp s}}}{q_{s}} \mathcal{G}_{20_{s}} \frac{\widetilde{B}_{\|}}{B_{0}}\right)-v_{t h_{\| s}}\left(\delta_{m 0}+\delta_{m 2}\right) \frac{q_{s}}{T_{0_{\| s}} c} \frac{\partial}{\partial z} \mathcal{G}_{10_{s}} \widetilde{A}_{\|}\right)=0
\end{aligned}
$$

for $0 \leq i \leq \mathcal{N}_{s}$ and $s=e, i$. In the two-dimensional (2D) limit in which the dependence on the $z$ coordinate is suppressed, the equations of motion (62) reduce, for a given species $s$, to

$$
\frac{\partial G_{i_{s}}}{\partial t}+\widetilde{\mathbf{v}}_{i_{s}} \cdot \nabla G_{i_{s}}=0, \quad 0 \leq i \leq \mathcal{N}_{s}
$$

where

$$
\widetilde{\mathbf{v}}_{i_{s}}=\hat{z} \times \nabla\left(\mathcal{G}_{10_{s}} \tilde{\phi}+\frac{2 T_{0_{\perp s}}}{q_{s}} \mathcal{G}_{20_{s}} \frac{\widetilde{B}_{\|}}{B_{0}}-\lambda_{i_{s}} \frac{v_{t h_{\| s}}}{c} \mathcal{G}_{10_{s}} \widetilde{A}_{\|}\right)
$$

This shows that, in the 2D limit, the system (57) can be cast in the form of advection equations for the Lagrangian invariants $G_{i_{s}}$, transported by the incompressible velocity fields $\widetilde{\mathbf{v}}_{i_{s}}$ defined in Eq. (64).

The Hamiltonian for the system (57), (52)-(54), on the other hand, is best expressed in terms of the original variables $g_{0 n_{s}}$ and is obtained [20] directly from the Hamiltonian 
of the parent model by replacing into Eq. (41), the following truncation of the expansion $(47)$ :

$$
g_{0_{s}}\left(\mathbf{x}, v_{\|}, t\right)=\sum_{n=0}^{\mathcal{N}_{s}} \frac{g_{0 n_{s}}(\mathbf{x}, t)}{\sqrt{n !}} H_{n}\left(\frac{v_{\|}}{v_{t h_{\| s}}}\right) F_{e q_{s}}\left(v_{\|}\right)
$$

The resulting Hamiltonian reads

$$
\begin{aligned}
& H\left(g_{00_{e}}, \cdots, g_{0 \mathcal{N}_{e e}}, g_{00_{i}}, \cdots, g_{0 \mathcal{N}_{i i}}\right) \\
& =\frac{1}{2} \sum_{s} n_{0} \int d^{3} x\left(T_{0_{\| s}} \sum_{n=0}^{\mathcal{N}_{s}} g_{0 n_{s}}^{2}+q_{s} g_{00_{s}}\left(\mathcal{G}_{10_{s}} \widetilde{\phi}+\frac{2 T_{0_{\perp s}}}{q_{s}} \mathcal{G}_{20_{s}} \frac{\widetilde{B}_{\|}}{B_{0}}\right)-q_{s} g_{01_{s}} \frac{v_{t h_{\| s}}}{c} \mathcal{G}_{10_{s}} \widetilde{A}_{\|}\right) .
\end{aligned}
$$

The Hamiltonian (66) can then be expressed in terms of the variables $G_{i_{s}}$. By inverting the relation (59) it is possible to express the gyrofluid moments $g_{0 n_{s}}$ in terms of the variables $G_{i_{s}}$. The resulting expression, combined with the Poisson bracket (60), yields the gyrofluid evolution equations (62). With regard to this last step, it is helpful to remark that, by means of the expression (61) and making use of the symmetry of the operators $\mathcal{G}_{10_{s}}, \mathcal{G}_{20_{s}}, \mathcal{L}_{\phi_{s}}, \mathcal{L}_{A_{s}}$ and $\mathcal{L}_{B_{s}}$, one finds that the functional derivatives of the Hamiltonian with respect to the fields $G_{i_{s}}$ are given by

$$
\frac{\delta H}{\delta G_{i_{s}}}=q_{s} n_{0} v_{\left(i_{s}\right)}\left(\mathcal{G}_{10_{s}} \widetilde{\phi}+\frac{2 T_{0_{\perp s}}}{q_{s}} \mathcal{G}_{20_{s}} \frac{\widetilde{B}_{\|}}{B_{0}}\right)-q_{s} n_{0} \lambda_{i_{s}} v_{\left(i_{s}\right)} \frac{v_{t h_{\| s}}}{c} \mathcal{G}_{10_{s}} \widetilde{A}_{\|},
$$

for $i=0, \cdots, \mathcal{N}_{s}$ and $s=e, i$.

Noncanonical Hamiltonian systems such as those belonging to the class of gyrofluid models we derived, are characterized by conservation laws associated with Casimir invariants. These are functionals $C$ of the field variables such that $\{F, C\}=0$, for all functionals $F$, and where $\{$,$\} is the Poisson bracket of the system. It follows,$ in particular, that $\partial_{t} C=\{C, H\}=0$, which justifies why such functionals $C$ are invariant. In the case of the gyrofluid systems (57), complemented by Eqs. (52)-(54), the identification of the Casimir invariants becomes simplest if carried out in terms of the variables $G_{i_{s}}$. Indeed, from the expression (60) for the Poisson bracket in terms of such variables, it is possible to see that the functionals

$$
C_{i_{s}}=\int d^{3} x G_{i_{s}}, \quad i=0, \cdots, \mathcal{N}_{s}, \quad s=e, i
$$


are Casimir invariants. In the 2D limit, in particular, infinite families of such invariants exist, and are given by

$$
C_{i_{s}}=\int d^{2} x \mathcal{C}_{i_{s}}\left(G_{i_{s}}\right), \quad i=0, \cdots, \mathcal{N}_{s}, \quad s=e, i
$$

where $\mathcal{C}_{i_{s}}$ are arbitrary functions. The existence of such infinite families is related to the possibility, offered by the $2 \mathrm{D}$ limit, of casting the system in the Lagrangian invariant form (63). This feature is common to many fluid and gyrofluid reduced models for plasmas $[21,20,30,15,13,14,31,16]$.

\section{Example: a five-field model}

In this Section we provide an example of Hamiltonian gyrofluid model that can be built by means of the above described procedure.

In this example we consider a model describing the evolution of the first two moments for the electron species (i.e. $g_{00_{e}}$ and $g_{01_{e}}$ ) and of the first three moments for the ion species (i.e. $g_{00_{i}}, g_{01_{i}}$ and $g_{02_{i}}$ ), which corresponds to setting $\mathcal{N}_{e}=1$ and $\mathcal{N}_{i}=2$. We close the hierarchy of Eqs. (51) by imposing that the gyrofluid electron parallel temperature and ion parallel heat flux fluctuations are both zero, i.e., $g_{02_{e}}=g_{03_{i}}=0$, which, from Eq. (56), amounts to setting $\alpha_{e}=\alpha_{i}=0$.

We express the model in terms of the following set of normalized variables, which provide a physically more transparent notation and make the connections with models already present in the literature easier:

$$
\begin{aligned}
& x=\frac{\widetilde{x}}{\rho_{s}}, \quad y=\frac{\widetilde{y}}{\rho_{s}}, \quad z=\frac{\widetilde{z}}{\rho_{s}}, \quad t=\omega_{c i} \widetilde{t}, \\
& \phi=\frac{e \widetilde{\phi}}{T_{0_{\perp e}}}, \quad A_{\|}=\frac{\widetilde{A}_{\|}}{B_{0} \rho_{s}}, \quad B_{\|}=\frac{\widetilde{B}_{\|}}{B_{0}}, \quad U_{s}=\frac{\widetilde{U_{s}}}{c_{s_{\perp}}}, \\
& N_{s}=g_{00_{s}}, \quad A_{s}=\operatorname{sgn}\left(q_{s}\right) \frac{m_{s}}{m_{i}} \frac{v_{t h_{\| s}}}{c_{s_{\perp}}} g_{01_{s}}, \quad T_{\|_{s}}=\sqrt{2} g_{02_{s}}
\end{aligned}
$$

where

$$
c_{s_{\perp}}=\sqrt{\frac{T_{0_{\perp e}}}{m_{i}}}, \quad \rho_{s}=\frac{c_{s_{\perp}}}{\omega_{c i}}
$$


Hamiltonian gyrofluid reductions of gyrokinetic equations

are referred to as sound speed and sonic Larmor radius, respectively, and are both related to the perpendicular electron equilibrium temperature. In Eqs. (70)-(72), the tilde symbol refers to dimensional quantities. We also remark that the explicit expressions for the dynamical variables $A_{e}$ and $A_{i}$, corresponding to gyrofluid versions of the electron and ion parallel canonical momenta, are given by

$$
A_{e}=\mathcal{G}_{10_{e}} A_{\|}-\delta^{2} U_{e}, \quad A_{i}=\mathcal{G}_{10_{i}} A_{\|}+U_{i}
$$

where $\delta^{2}=m_{e} / m_{i}$ is the mass ratio.

Taking into account these considerations, the resulting five-field model consists of the following five evolution equations, obtained from truncating Eqs. (57),

$$
\begin{aligned}
& \frac{\partial N_{e}}{\partial t}+\left[\mathcal{G}_{10_{e}} \phi-2 \mathcal{G}_{20_{e}} B_{\|}, N_{e}\right]-\left[\mathcal{G}_{10_{e}} A_{\|}, U_{e}\right]+\frac{\partial U_{e}}{\partial z}=0 \\
& \frac{\partial}{\partial t}\left(\mathcal{G}_{10_{e}} A_{\|}-\delta^{2} U_{e}\right)+\left[\mathcal{G}_{10_{e}} \phi-2 \mathcal{G}_{20_{e}} B_{\|}, \mathcal{G}_{10_{e}} A_{\|}-\delta^{2} U_{e}\right]+\frac{1}{\Theta_{e}}\left[\mathcal{G}_{10_{e}} A_{\|}, N_{e}\right] \\
& +\frac{\partial}{\partial z}\left(\mathcal{G}_{10_{e}} \phi-2 \mathcal{G}_{20_{e}} B_{\|}-\frac{N_{e}}{\Theta_{e}}\right)=0, \\
& \frac{\partial N_{i}}{\partial t}+\left[\mathcal{G}_{10_{i}} \phi+2 \tau_{\perp_{i}} \mathcal{G}_{20_{i}} B_{\|}, N_{i}\right]-\left[\mathcal{G}_{10_{i}} A_{\|}, U_{i}\right]+\frac{\partial U_{i}}{\partial z}=0 \\
& \frac{\partial}{\partial t}\left(\mathcal{G}_{10_{i}} A_{\|}+U_{i}\right)+\left[\mathcal{G}_{10_{i}} \phi+2 \tau_{\perp_{i}} \mathcal{G}_{20_{i}} B_{\|}, \mathcal{G}_{10_{i}} A_{\|}+U_{i}\right]-\frac{\tau_{\perp_{i}}}{\Theta_{i}}\left[\mathcal{G}_{10_{i}} A_{\|}, N_{i}+T_{\|_{i}}\right] \\
& +\frac{\partial}{\partial z}\left(\mathcal{G}_{10_{i}} \phi+2 \tau_{\perp_{i}} \mathcal{G}_{20_{i}} B_{\|}+\frac{N_{i}}{\Theta_{i}}\right)=0, \\
& \frac{\partial T_{\|_{i}}}{\partial t}+\left[\mathcal{G}_{10_{i}} \phi+2 \tau_{\perp_{i}} \mathcal{G}_{20_{i}} B_{\|}, T_{\|_{i}}\right]-2\left[\mathcal{G}_{10_{i}} A_{\|}, U_{i}\right]+2 \frac{\partial U_{i}}{\partial z}=0
\end{aligned}
$$

complemented by the relations

$$
\begin{aligned}
& \mathcal{G}_{10_{i}} N_{i}-\mathcal{G}_{10_{e}} N_{e}=\left(\frac{1-\Gamma_{0}\left(b_{i}\right)}{\tau_{\perp_{i}}}+1-\Gamma_{0}\left(b_{e}\right)\right) \phi-\left(\Gamma_{0}\left(b_{i}\right)-\Gamma_{1}\left(b_{i}\right)-\Gamma_{0}\left(b_{e}\right)+\Gamma_{1}\left(b_{e}\right)\right) B_{\|} \\
& \mathcal{G}_{10_{e}} U_{e}-\mathcal{G}_{10_{i}} U_{i}=\frac{2}{\beta_{\perp_{e}}} \nabla_{\perp}^{2} A_{\|}+\left(\left(1-\frac{1}{\Theta_{i}}\right)\left(\Gamma_{0}\left(b_{i}\right)-1\right)+\left(1-\frac{1}{\Theta_{e}}\right) \frac{\Gamma_{0}\left(b_{e}\right)-1}{\delta^{2}}\right) A_{\|} \\
& 2 \tau_{\perp_{i}} \mathcal{G}_{20_{i}} N_{i}+2 \mathcal{G}_{20_{e}} N_{e}= \\
& -2\left(\frac{1}{\beta_{\perp_{e}}}+\Gamma_{0}\left(b_{e}\right)-\Gamma_{1}\left(b_{e}\right)+\tau_{\perp_{i}}\left(\Gamma_{0}\left(b_{i}\right)-\Gamma_{1}\left(b_{i}\right)\right)\right) B_{\|}+\left(\Gamma_{0}\left(b_{e}\right)-\Gamma_{1}\left(b_{e}\right)-\Gamma_{0}\left(b_{i}\right)+\Gamma_{1}\left(b_{i}\right)\right) \phi
\end{aligned}
$$


descending from Eqs. (52)-(54). This model generalizes previous gyrofluid models such as those of Refs. $[16,14,13]$ in the absence of equilibrium magnetic curvature and density gradients (which could be added a posteriori), by including parallel magnetic fluctuations, equilibrium temperature anisotropy and electron FLR effects. In particular, by including the terms related to magnetic curvature, the model could be used for instance to extend the analysis of the Ion Temperature Gradient instability performed in Ref. [16].

By construction, the model (75)-(79),(80)-(82) is Hamiltonian. The Hamiltonian functional corresponds to (66) and, in the normalized variables, it reads

$$
\begin{aligned}
& H\left(N_{e}, N_{i}, A_{e}, A_{i}, T_{\|_{i}}\right) \\
& =\frac{1}{2} \int d^{3} x\left(\frac{N_{e}^{2}}{\Theta_{e}}+\frac{\tau_{\perp_{i}}}{\Theta_{i}} N_{i}^{2}+\frac{A_{e}^{2}}{\delta^{2}}+A_{i}^{2}+\frac{\tau_{\perp_{i}}}{\Theta_{i}} \frac{T_{\|_{i}}^{2}}{2}\right. \\
& \left.+N_{i}\left(\mathcal{G}_{10_{i}} \phi+2 \tau_{\perp_{i}} \mathcal{G}_{20_{i}} B_{\|}\right)-N_{e}\left(\mathcal{G}_{10_{e}} \phi-2 \mathcal{G}_{20_{e}} B_{\|}\right)-A_{i} \mathcal{G}_{10_{i}} A_{\|}-\frac{A_{e}}{\delta^{2}} \mathcal{G}_{10_{e}} A_{\|}\right) .
\end{aligned}
$$

Given that, for the five-field model under consideration, $\alpha_{e}=\alpha_{i}=0$, from Eq. (58) we obtain that the expressions for the matrices $W_{e}$ and $W_{i}$ are given by

$$
W_{e}=\left(\begin{array}{cc}
0 & 1 \\
1 & 0
\end{array}\right), \quad W_{i}=\left(\begin{array}{ccc}
0 & 1 & 0 \\
1 & 0 & \sqrt{2} \\
0 & \sqrt{2} & 0
\end{array}\right)
$$

and the corresponding eigenvalues read

$$
\begin{aligned}
& \lambda_{0_{e}}=1, \quad \lambda_{1_{e}}=-1, \\
& \lambda_{0_{i}}=\sqrt{3}, \quad \lambda_{1_{i}}=-\sqrt{3}, \quad \lambda_{2_{i}}=0 .
\end{aligned}
$$

The orthogonal matrices $\mathcal{U}_{e}$ and $\mathcal{U}_{i}$, on the other hand, correspond to

$$
\mathcal{U}_{e}=\left(\begin{array}{cc}
\frac{1}{\sqrt{2}} & \frac{1}{\sqrt{2}} \\
\frac{1}{\sqrt{2}} & -\frac{1}{\sqrt{2}}
\end{array}\right), \quad \mathcal{U}_{i}=\left(\begin{array}{ccc}
\frac{1}{\sqrt{6}} & \frac{1}{\sqrt{6}} & -\sqrt{\frac{2}{3}} \\
\frac{1}{\sqrt{2}} & -\frac{1}{\sqrt{2}} & 0 \\
\frac{1}{\sqrt{3}} & \frac{1}{\sqrt{3}} & \frac{1}{\sqrt{3}}
\end{array}\right)
$$


Hamiltonian gyrofluid reductions of gyrokinetic equations

Consequently, the alternative set of variables $\left\{G_{0_{e}}, G_{1_{e}}, G_{0_{i}}, G_{1_{i}}, G_{2_{i}}\right\}$ is given by

$$
\begin{aligned}
G_{0_{e}} & =\frac{g_{00_{e}}}{\sqrt{2}}+\frac{g_{01_{e}}}{\sqrt{2}}, \quad G_{1_{e}}=\frac{g_{00_{e}}}{\sqrt{2}}-\frac{g_{01_{e}}}{\sqrt{2}} \\
G_{0_{i}} & =\frac{g_{00_{i}}}{\sqrt{6}}+\frac{g_{01_{i}}}{\sqrt{2}}+\frac{g_{02_{i}}}{\sqrt{3}}, \quad G_{1_{i}}=\frac{g_{00_{i}}}{\sqrt{6}}-\frac{g_{01_{i}}}{\sqrt{2}}+\frac{g_{02_{i}}}{\sqrt{3}}, \\
G_{2_{i}} & =-\sqrt{\frac{2}{3}} g_{00_{i}}+\frac{g_{02_{i}}}{\sqrt{3}}
\end{aligned}
$$

or, in terms of the variables (70)-(72),

$$
\begin{aligned}
G_{0_{e}} & =\frac{N_{e}}{\sqrt{2}}-\sqrt{\frac{\Theta_{e}}{2}} \frac{A_{e}}{\delta}, \quad G_{1_{e}}=\frac{N_{e}}{\sqrt{2}}+\sqrt{\frac{\Theta_{e}}{2}} \frac{A_{e}}{\delta} \\
G_{0_{i}} & =\frac{N_{i}}{\sqrt{6}}+\sqrt{\frac{\Theta_{i}}{2 \tau_{\perp_{i}}}} A_{i}+\frac{T_{\|_{i}}}{\sqrt{6}}, \quad G_{1_{i}}=\frac{N_{i}}{\sqrt{6}}-\sqrt{\frac{\Theta_{i}}{2 \tau_{\perp_{i}}}} A_{i}+\frac{T_{\|_{i}}}{\sqrt{6}}, \\
G_{2_{i}} & =-\sqrt{\frac{2}{3}} N_{i}+\frac{T_{\|_{i}}}{\sqrt{6}} .
\end{aligned}
$$

According to Eq. (68), the Casimir invariants of the model are given by

$$
\begin{aligned}
C_{0_{e}} & =\int d^{3} x G_{0_{e}}, & C_{1_{e}} & =\int d^{3} x G_{1_{e}}, \\
C_{0_{i}} & =\int d^{3} x G_{0_{i}}, & C_{1_{i}} & =\int d^{3} x G_{1_{i}},
\end{aligned}
$$

In the 2D limit, following Eq. (69), the model admits five infinite families of Casimir invariants, corresponding to

$$
\begin{aligned}
C_{m_{e}}=\int d^{2} x \mathcal{C}_{m_{e}}\left(G_{m_{e}}\right), & m=0,1, \\
C_{n_{i}}=\int d^{2} x \mathcal{C}_{n_{i}}\left(G_{n_{i}}\right), & n=0,1,2,
\end{aligned}
$$

with $\mathcal{C}_{0,1_{e}}$ and $\mathcal{C}_{0,1,2_{i}}$ arbitrary functions.

Also, from Eq. (63), it emerges that, in 2D, the system can be cast in the following form of advection equations

$$
\begin{aligned}
& \frac{\partial G_{m_{e}}}{\partial t}+\mathbf{v}_{m_{e}} \cdot \nabla G_{m_{e}}=0, \\
& \frac{\partial G_{n_{i}}}{\partial t}+\mathbf{v}_{n_{i}} \cdot \nabla G_{n_{i}}=0,
\end{aligned}
$$


where

$$
\begin{aligned}
& \mathbf{v}_{0_{e}}=\hat{z} \times \nabla\left(\mathcal{G}_{10_{e}} \phi-2 \mathcal{G}_{20_{e}} B_{\|}-\frac{\mathcal{G}_{10_{e}} A_{\|}}{\delta \sqrt{\Theta_{e}}}\right), \\
& \mathbf{v}_{1_{e}}=\hat{z} \times \nabla\left(\mathcal{G}_{10_{e}} \phi-2 \mathcal{G}_{20_{e}} B_{\|}+\frac{\mathcal{G}_{10_{e}} A_{\|}}{\delta \sqrt{\Theta_{e}}}\right), \\
& \mathbf{v}_{0_{i}}=\hat{z} \times \nabla\left(\mathcal{G}_{10_{i}} \phi+2 \tau_{\perp_{i}} \mathcal{G}_{20_{i}} B_{\|}-\sqrt{\frac{3 \tau_{\perp_{i}}}{\Theta_{i}}} \mathcal{G}_{10_{i}} A_{\|}\right), \\
& \mathbf{v}_{1_{i}}=\hat{z} \times \nabla\left(\mathcal{G}_{10_{i}} \phi+2 \tau_{\perp_{i}} \mathcal{G}_{20_{i}} B_{\|}+\sqrt{\frac{3 \tau_{\perp_{i}}}{\Theta_{i}}} \mathcal{G}_{10_{i}} A_{\|}\right), \\
& \mathbf{v}_{2_{i}}=\hat{z} \times \nabla\left(\mathcal{G}_{10_{i}} \phi+2 \tau_{\perp_{i}} \mathcal{G}_{20_{i}} B_{\|}\right),
\end{aligned}
$$

which follows from Eq. (64) upon using the normalized variables (70)-(72). Compared to the models of Refs. $[16,14]$, one notices that the presence of parallel magnetic perturbations affects the stream functions associated with the velocity fields that advect the Lagrangian invariants. The presence of equilibrium temperature anisotropy affects the stream functions too. In particular, from Eqs. (100)-(103), one sees that, depending on whether the perpendicular temperature dominates over the parallel one or not, the contribution to the generalized velocity fields due to the perpendicular magnetic fluctuations (associated with $A_{\|}$) becomes less or more relevant.

\section{Remarks about variants of the procedure}

The procedure for deriving Hamiltonian gyrofluid models described in Sec. 4 admits some possible variants that might be helpful, should one be interested in incorporating specific physical effects into the model. On one hand, it is possible to consider expressions for the gyroaverage operators $\mathcal{G}_{1_{e, i}}$ and $\mathcal{G}_{2_{e, i}}$ different from those that follow from Eqs. (31)-(32). In the latter case, due to the assumed expansions (24) and (47), it follows that

$$
\mathcal{G}_{10_{s}}=\exp \left(-b_{s} / 2\right), \quad \mathcal{G}_{20_{s}}=\frac{\exp \left(-b_{s} / 2\right)}{2}
$$

This is in agreement with Ref. [4]. However, in the plasma physics literature, alternative choices for the expressions of the gyroaverage operators are also frequently used (see, e.g. Refs. $[5,9,11])$, in particular if a better agreement with the linear kinetic theory is 
prior. In the procedure described in Sec. 4 no use was made of the explicit expression for the operators $\mathcal{G}_{10_{e, i}}$ and $\mathcal{G}_{20_{e, i}}$ (the only ones appearing in the final gyrofluid models). In particular, for the resulting models to be Hamiltonian, it was not required that such gyroaverage operators have the expressions (105). The important property is that the gyroaverage operators be symmetric, i.e. such that $\int d^{3} x f \mathcal{G}_{10_{s}} g=\int d^{3} x g \mathcal{G}_{10_{s}} f$ and $\int d^{3} x f \mathcal{G}_{20_{s}} g=\int d^{3} x g \mathcal{G}_{20_{s}} f$ for two functions $f$ and $g$ and for $s=e, i$. Provided that this requirement is satisfied, linear differential operators (independent on the $v_{\|}$and $\mu_{0 s}$ coordinate) other than those of Eq. (105) can be used, if so wished, in order to derive Hamiltonian gyrofluid models with the above procedure.

A second variant concerns the relations (55) between electromagnetic quantities and gyrofluid moments. Also in this case, the above procedure can in principle be applied for operators $\mathcal{L}_{\phi_{s}}, \mathcal{L}_{A_{s}}$ and $\mathcal{L}_{B_{s}}$ other than those obtained by inverting the relations (52)-(54). It suffices that the (typically integral) operators $\mathcal{L}_{\phi_{s}}, \mathcal{L}_{A_{s}}$ and $\mathcal{L}_{B_{s}}$ be linear, symmetric, invertible and independent on the coordinates $v_{\|}$and $\mu_{0 s}$.

In order to exemplify a variant of the procedure described in Sec 4, we derive a twofield model that generalizes, by including equilibrium electron temperature anisotropy, the two-field model for kinetic Alfvén waves presented in Ref. [31].

The model describes the evolution of only the first two moments of the electron species, so that $s=e$ and $\mathcal{N}_{e}=1$. The closure on the parallel temperature fluctuations is again isothermal so that $\alpha_{e}=0$. Electron gyroaverage effects are neglected, assuming $b_{e} \ll 1$. The following expressions for the gyroaverage operators are then taken:

$$
\mathcal{G}_{10_{e}}=1, \quad \mathcal{G}_{20_{e}}=\frac{1}{2}
$$

which, of course, trivially satisfy the properties mentioned at the beginning of Sec. 6 .

Following these prescriptions, from Eq. (57), we obtain that the two evolution 
equations of the model are given by

$$
\begin{aligned}
& \frac{\partial N_{e}}{\partial t}+\left[\phi-B_{\|}, N_{e}\right]-\left[A_{\|}, U_{e}\right]+\frac{\partial U_{e}}{\partial z}=0 \\
& \frac{\partial}{\partial t}\left(A_{\|}-\delta^{2} U_{e}\right)+\left[\phi-B_{\|}, A_{\|}-\delta^{2} U_{e}\right]+\frac{1}{\Theta_{e}}\left[A_{\|}, N_{e}\right] \\
& +\frac{\partial}{\partial z}\left(\phi-B_{\|}-\frac{N_{e}}{\Theta_{e}}\right)=0
\end{aligned}
$$

On the other hand, the relations (55) for this model follow from

$$
\begin{aligned}
& N_{e}+\left(1-\Gamma_{0}\left(b_{i}\right)+\Gamma_{1}\left(b_{i}\right)\right) B_{\|}+\left(1-\Gamma_{0}\left(b_{i}\right)-\tau_{\perp_{i}} \delta^{2} \nabla_{\perp}^{2}\right) \frac{\phi}{\tau_{\perp_{i}}}=0, \\
& U_{e}=b_{*} \nabla_{\perp}^{2} A_{\|}, \\
& B_{\|}=-\frac{\beta_{\perp_{e}}}{2}\left(N_{e}-\left(1-\Gamma_{0}\left(b_{i}\right)+\Gamma_{1}\left(b_{i}\right)\right) \phi+\left(1+2 \tau_{\perp_{i}}\left(\Gamma_{0}\left(b_{i}\right)-\Gamma_{1}\left(b_{i}\right)\right)\right) B_{\|}\right),
\end{aligned}
$$

which can be obtained from Eqs. (2)-(4) by applying the ordering specified in Ref. [31] and allowing for equilibrium electron temperature anisotropy (note that, in order to derive Eq. (110) from Eq. (81), an expansion in the limit $\delta^{2} \rightarrow 0$ was performed, which is consistent with the limit $b_{e} \rightarrow 0$ yielding Eq. (106)).

In Eq. (110) the parameter

$$
b_{*}=\frac{2}{\beta_{\perp_{e}}}+1-\frac{1}{\Theta_{e}}
$$

was introduced, which clearly reduces to $2 / \beta_{\perp_{e}}$ in the absence of electron temperature anisotropy. We remark that the inequality $b_{*}<0$ corresponds to the condition for firehose instability (see, e.g. Ref. [32]).

Ion gyrofluid moments do not appear in the relations (109)-(111), (according to Ref. [31], in this model $N_{i}=T_{\|_{i}}=0$ and the evolution of $U_{i}$ is decoupled) so that the relations (55), in normalized variables, reduce to

$$
\phi=\frac{e}{T_{0_{\perp e}}} \mathcal{L}_{\phi_{e}} N_{e}, \quad A_{\|}=-\frac{c_{s_{\perp}}}{\delta^{2} v_{t h_{\| e}}} \frac{\mathcal{L}_{A_{e}}}{B_{0} \rho_{s}} A_{e}, \quad B_{\|}=\frac{\mathcal{L}_{B_{e}}}{B_{0}} N_{e}
$$

with the operators $\mathcal{L}_{\phi_{e}}, \mathcal{L}_{A_{e}}$ and $\mathcal{L}_{B_{e}}$ satisfying the required above mentioned properties. Ion gyroaverage effects, on the other hand, are taken into account and are associated with the functions $\Gamma_{0}\left(b_{i}\right)$ and $\Gamma_{1}\left(b_{i}\right)$. 
We remark that the relation (111) cannot be obtained as an asymptotic limit of Eq. (54) (or, in normalized form, of Eq. (82)). In particular, the factor 1, corresponding to the first term of the three terms multiplying $B_{\|}$on the right-hand side of Eq. (111), cannot be obtained from Eq. (54). Its origin comes from the closure assumption of Ref. [31], according to which the fluctuations of the perpendicular electron temperature, denoted, in normalized form, with $T_{\perp e}$, satisfy the relation $T_{\perp e}=-B_{\|}$, corresponding to an isothermal closure on the (particle, instead of gyrocenter) perpendicular temperature fluctuations as well. This closure is not compatible with the closure assumption (39) adopted in our procedure, and which concerns the moments with respect to the perpendicular velocity. More explicitly, the gyrocenter perpendicular electron temperature fluctuations are defined by

$$
\begin{aligned}
& T_{\perp e}(\mathbf{x}, t)=\frac{1}{n_{0}} \int d \mathcal{W}_{e}\left(\frac{\mu_{0 e} B_{0}}{T_{0_{\perp e}}}-1\right) \tilde{f}_{e}\left(\mathbf{x}, v_{\|}, \mu_{0 e}, t\right) \\
& =-\frac{1}{n_{0}} \int d \mathcal{W}_{e} L_{1}\left(\frac{\mu_{0 e} B_{0}}{T_{0_{\perp e}}}\right) \mathrm{f}_{e q_{e}}\left(\mu_{0 e}\right) \sum_{n=0}^{+\infty} L_{n}\left(\frac{\mu_{0 e} B_{0}}{T_{0_{\perp e}}}\right) f_{n_{e}}\left(\mathbf{x}, v_{\|}, t\right)=-\frac{1}{n_{0}} \int d v_{\|} f_{1_{e}}\left(\mathbf{x}, v_{\|}, t\right),
\end{aligned}
$$

where use was made of the expansion (27) and of the orthogonality of Laguerre polynomials. According to our closure (39), we would have $f_{1_{e}}=0$, which implies $T_{\perp e}=0$. This is evidently in contrast with the closure $T_{\perp e}=-B_{\|}$used to derive Eq. (111) from Eq. (4). Nevertheless, because the Equations (109)-(111) still lead to relations of the form (113), a Hamiltonian gyrofluid model can be derived. This shows that, in principle, our procedure can be extended to include also relations between electromagnetic fields and gyrofluid moments different from those descending from Eqs. (52)-(54)

The Hamiltonian functional of the model, obtained from (66), reads

$$
H\left(N_{e}, A_{e}\right)=\frac{1}{2} \int d^{3} x\left(\frac{N_{e}^{2}}{\Theta_{e}}+\frac{A_{e}^{2}}{\delta^{2}}-N_{e}\left(\phi-B_{\|}\right)-\frac{A_{e}}{\delta^{2}} A_{\|}\right) .
$$

Making use of Eq. (110) and of the relation $A_{e}=A_{\|}-\delta^{2} U_{e}$, the Hamiltonian (116) can 
be rewritten as

$$
H\left(N_{e}, A_{e}\right)=\frac{1}{2} \int d^{3} x\left(\frac{N_{e}^{2}}{\Theta_{e}}+\delta^{2} U_{e}^{2}+b_{*}\left|\nabla_{\perp} A\right|^{2}-N_{e}\left(\phi-B_{\|}\right)\right),
$$

which lets emerge the contribution due to the parallel kinetic energy and to the perpendicular magnetic energy, modified by magnetization effects due to the temperature anisotropy. These two contributions correspond to the second and third term of Eq. (117), respectively.

Finally, we remark that, because $\mathcal{N}_{e}=1$ and $\alpha_{e}=0$, as in the five-field model of Sec. 5, the matrices $\mathcal{W}_{e}$ and $\mathcal{U}_{e}$ are again those appearing in Eqs. (84) and (87). Likewise, the expressions for the alternative variables $G_{0_{e}}$ and $G_{1_{e}}$, as well as for the Casimir invariants and for the 2D formulation in terms of Lagrangian invariants are analogous (provided, of course, that the form (106) is taken for the gyroaverage operators) to those for the two electron moments of the five-field model and correspond to Eqs. (91), (94) and (98), respectively.

\section{Conclusions}

We presented a framework based on a Hamiltonian reduction, which makes it possible to derive Hamiltonian gyrofluid models for an arbitrary number of moments in the parallel velocity coordinate. The resulting models include, in addition to finite Larmor radius effects, magnetic perturbations along the equilibrium magnetic field and equilibrium temperature anisotropies, which are not present in Hamiltonian gyrofluid models available in the literature. The Hamiltonian reduction is presented in two stages. A first reduction leads from the original Hamiltonian gyrokinetic model (1)-(4), evolving the variables $\widetilde{g}_{s}\left(\mathbf{x}, v_{\|}, \mu_{0 s}, t\right)$ (or, equivalently, the variables $\widetilde{f}_{s}\left(\mathbf{x}, v_{\|}, \mu_{0 s}, t\right)$ ) to the Hamiltonian model (46), (42)-(44) evolving the averaged perturbation of the distribution function $g_{0_{s}}\left(\mathbf{x}, v_{\|}, t\right)$ (or $f_{0_{s}}\left(\mathbf{x}, v_{\|}, t\right)$ ) depending on a reduced number of coordinates. The second stage applies a closure to the system (46), (42)-(44) yielding Hamiltonian gyrofluid models (57), (52)-(54), evolving the moment variables $g_{0 m_{s}}(\mathbf{x}, t)\left(\right.$ or $\left.f_{0 m_{s}}(\mathbf{x}, t)\right)$, for $0 \leq m \leq \mathcal{N}_{s}$, with arbitrary $\mathcal{N}_{s}$. Casimir invariants for the Hamiltonian gyrofluid 
models were presented and it was shown that, in the two-dimensional limit, they can be cast in form of systems of advection equations for Lagrangian invariants (associated with the Casimir invariants), transported by generalized incompressible flows. The fivefield model presented in Sec. 5 and the two-field model presented in Sec. 6, permit to observe the features of the general models by means of concrete examples.

The analysis of the physical properties of the gyrofluid models derived by means of the above procedure is an on-going work. In particular, as anticipated in Sec. 1, the inclusion of finite $\beta$ effects and of equilibrium temperature anisotropy make these models suitable for application to space plasmas. For instance, they could be applied for the investigation of instabilities, such the firehose or the swelling instability, induced by equilibrium temperature anisotropy and relevant for the solar wind. A comparison with the results obtained from the linearized parent gyrokinetic system, analyzed in Ref. [33], would also be a natural step in order to identify advantages and limitations of the reduced gyrofluid models with respect to the original gyrokinetic model.

We remark that the gyrofluid models considered in the present paper are derived from a gyrokinetic system evolving small perturbations of the equilibrium distribution functions. A natural step forward would be to identify Hamiltonian reductions of "fullf" gyrokinetic systems (such as that adopted, for instance, in Ref. [12]), following the direction of Refs. [26, 34], where Hamiltonian closures for drift-kinetic models were derived. The additional nonlinearities present in such systems, however, makes the treatment of the electromagnetic problem considerably more complex.

With regard to more mathematical aspects, an obvious question concerns the possibility to find Hamiltonian reductions leading to gyrofluid models evolving also finite order moments with respect to the coordinate $\mu_{0 s}$. Hamiltonian reduced models evolving also such moments were presented in Refs. [35, 36, 37] but did not take into account finite Larmor radius effects when both parallel and perpendicular temperature fluctuations for the same species were considered. In Ref. [11] an energy conserving gyrofluid model including both parallel and perpendicular temperature as well as heat 
flux fluctuations is presented, but its Hamiltonian structure has not been found yet. The presence of gyroaverage operators associated with the functions $J_{0}$ and $J_{1}$, depending on $\mu_{0 s}$, in the parent gyrokinetic system, makes the gyrofluid moment equations depend in principle on an infinite number of moments with respect to the $\mu_{0 s}$ coordinates. This provides a significant difference with respect to the hierarchy of equations (51) obtained by taking moments only with respect to the $v_{\|}$coordinate. Identifying sub-algebras other than the trivial one adopted in this paper, involving functionals of moments with respect to polynomials in $\mu_{0 s}$, appears not to be an easy task and is part of future work.

\section{Acknowledgments}

ET acknowledges useful discussions with Thierry Passot and Pierre-Louis Sulem. This material is based upon work supported by the National Science Foundation under Grant No. 1440140, while the author was in residence at the Mathematical Sciences Research Institute in Berkeley, California, during the Fall semester of year 2018.

\section{Appendix A. The invertibility of the operators acting on $\widetilde{\phi}, \widetilde{A}_{\|}$and $\widetilde{B}_{\|}$in the quasi-neutrality relation and in Ampère's law}

The quasi-neutrality relation (2), the parallel (3) and perpendicular (4) Ampère's law

relate the electromagnetic perturbations $\widetilde{\phi}, \widetilde{A}_{\|}$and $\widetilde{B}_{\|}$to the functions $\widetilde{g}_{e}$ and $\widetilde{g}_{i}$. In order for the fields $\widetilde{\phi}, \widetilde{A}_{\|}$and $\widetilde{B}_{\|}$to be well defined, it is necessary that such fields can be expressed in the form

$$
\begin{aligned}
& \widetilde{\phi}=L_{\phi_{e}} \widetilde{g}_{e}+L_{\phi_{i}} \widetilde{g}_{i}, \\
& \widetilde{A}_{\|}=L_{A_{e}} \widetilde{g}_{e}+L_{A_{i}} \widetilde{g}_{i}, \\
& \widetilde{B}_{\|}=L_{B_{e}} \widetilde{g}_{e}+L_{B_{i}} \widetilde{g}_{i},
\end{aligned}
$$

for some operators $L_{\phi_{e, i}}, L_{A_{e, i}}, L_{B_{e, i}}$. Moreover, the expression (21) for the functional derivative of the gyrokinetic Hamiltonian follows from the symmetry of the operators $\mathcal{J}_{i_{s s^{\prime}}}$, for $i=1,2,3$, which in turn depends on the form of the operators $L_{\phi_{e, i}}, L_{A_{e, i}}, L_{B_{e, i}}$. 
These issues turn out to be rather simple to handle under our assumption of spatial domain with periodic boundary conditions, and are discussed hereafter.

Namely by virtue of the hypothesis of periodicity, we perform the following Fourier series expansions in the spatial coordinates:

$$
\begin{aligned}
& \widetilde{g}_{s}\left(\mathbf{x}, v_{\|}, \mu_{0 s}, t\right)=\sum_{\mathbf{k} \in \mathscr{D}} \widetilde{g}_{s \mathbf{k}}\left(v_{\|}, \mu_{0 s}, t\right) \mathrm{e}^{i \mathbf{k} \cdot \mathbf{x}} \\
& \widetilde{\phi}(\mathbf{x}, t)=\sum_{\mathbf{k} \in \mathscr{D}} \widetilde{\phi}_{\mathbf{k}}(t) \mathrm{e}^{i \mathbf{k} \cdot \mathbf{x}}, \quad \widetilde{B}_{\|}(\mathbf{x}, t)=\sum_{\mathbf{k} \in \mathscr{D}} \widetilde{B}_{\| \mathbf{k}}(t) \mathrm{e}^{i \mathbf{k} \cdot \mathbf{x}}, \\
& \widetilde{A}_{\|}(\mathbf{x}, t)=\sum_{\mathbf{k} \in \mathscr{D}} \widetilde{A}_{\| \mathbf{k}}(t) \mathrm{e}^{i \mathbf{k} \cdot \mathbf{x}} .
\end{aligned}
$$

For each wave vector $\mathbf{k}$, the linear relations (2) and (4) yield

$$
\begin{aligned}
& M_{e \mathbf{k}} \widetilde{g}_{e \mathbf{k}}+M_{i \mathbf{k}} \widetilde{g}_{i \mathbf{k}}=M_{\phi \mathbf{k}} \widetilde{\phi}_{\mathbf{k}}+M_{B \mathbf{k}} \widetilde{B}_{\| \mathbf{k}}, \\
& N_{e \mathbf{k}} \widetilde{g}_{e \mathbf{k}}+N_{i \mathbf{k}} \widetilde{g}_{i \mathbf{k}}=N_{\phi \mathbf{k}} \widetilde{\phi}_{\mathbf{k}}+N_{B \mathbf{k}} \widetilde{B}_{\| \mathbf{k}}
\end{aligned}
$$

respectively, where the Fourier multipliers $M_{s \mathbf{k}}, M_{\phi \mathbf{k}}, M_{B \mathbf{k}}, N_{s \mathbf{k}}, N_{\phi \mathbf{k}}, N_{B \mathbf{k}}$ are defined by $M_{s \mathbf{k}}=\int d \mathcal{W}_{s} J_{0}\left(a_{s}\right), \quad N_{s \mathbf{k}}=\frac{\beta_{\perp_{s}}}{n_{0}} \int d \mathcal{W}_{s} 2 \frac{\mu_{0 s} B_{0}}{T_{0_{\perp s}}} \frac{J_{1}\left(a_{s}\right)}{a_{s}}$,
$M_{\phi \mathbf{k}}=n_{0} \sum_{s} \frac{\left|q_{s}\right|}{T_{0_{\perp s}}}\left(1-\Gamma_{0}\left(b_{s}\right)\right), \quad N_{\phi \mathbf{k}}=-\sum_{s} \beta_{\perp_{s}} \frac{q_{s}}{T_{0_{\perp s}}}\left(\Gamma_{0}\left(b_{s}\right)-\Gamma_{1}\left(b_{s}\right)\right)$,
$M_{B \mathbf{k}}=-\frac{n_{0}}{B_{0}} \sum_{s} \operatorname{sgn}\left(q_{s}\right)\left(\Gamma_{0}\left(b_{s}\right)-\Gamma_{1}\left(b_{s}\right)\right), \quad N_{B \mathbf{k}}=-\frac{2}{B_{0}}\left(1+\sum_{s} \beta_{\perp_{s}}\left(\Gamma_{0}\left(b_{s}\right)-\Gamma_{1}\left(b_{s}\right)\right)\right)$.

On the other hand, Eq. (3) yields

$$
O_{e \mathbf{k}} \widetilde{g}_{e \mathbf{k}}+O_{i \mathbf{k}} \widetilde{g}_{i \mathbf{k}}=O_{A \mathbf{k}} \widetilde{A}_{\| \mathbf{k}}
$$

where

$$
\begin{aligned}
O_{s \mathbf{k}} & =q_{s} \int d \mathcal{W}_{s} v_{\|} J_{0}\left(a_{s}\right) \\
O_{A \mathbf{k}} & =\sum_{s} \frac{q_{s}^{2} n_{0}}{m_{s} c} \Gamma_{0}\left(b_{s}\right)+\frac{c}{4 \pi} k_{\perp}^{2}+\sum_{s} \frac{q_{s}^{2} n_{0}}{m_{s} c}\left(1-\frac{1}{\Theta_{s}}\right)\left(1-\Gamma_{0}\left(b_{s}\right)\right)
\end{aligned}
$$

We begin by discussing the invertibility of the operators appearing in the system (A.7)(A.8), which involves $\widetilde{\phi}_{\mathbf{k}}$ and $\widetilde{B}_{\| \mathbf{k}}$. 
Hamiltonian gyrofluid reductions of gyrokinetic equations

Because the operators $M_{\phi \mathbf{k}}, M_{B \mathbf{k}}, N_{\phi \mathbf{k}}$ and $N_{B \mathbf{k}}$ all consist of linear combinations of multiplication operators in Fourier space, the invertibility condition amounts, for each $\mathbf{k}$, to the determinant of the matrix

$$
\mathcal{Q}_{\mathbf{k}}=\left(\begin{array}{cc}
M_{\phi \mathbf{k}} & M_{B \mathbf{k}} \\
N_{\phi \mathbf{k}} & N_{B \mathbf{k}}
\end{array}\right)
$$

not to be zero. Such determinant is given by

$$
\begin{aligned}
& \operatorname{det} \mathcal{Q}_{\mathbf{k}}=-2 \frac{n_{0}}{B_{0}} e \sum_{s} \frac{1-\Gamma_{0}\left(b_{s}\right)}{T_{0_{\perp s}}}\left(1+\sum_{s^{\prime}} \beta_{\perp_{s}}\left(\Gamma_{0}\left(b_{s^{\prime}}\right)-\Gamma_{1}\left(b_{s^{\prime}}\right)\right)\right) \\
& -8 \pi \frac{e n_{0}^{2}}{B_{0}^{3}}\left(\sum_{s}\left(\Gamma_{0}\left(b_{s}\right)-\Gamma_{1}\left(b_{s}\right)\right)\right)^{2}<0 .
\end{aligned}
$$

Indeed, the first term on the right-hand side of Eq.(A.16) is always negative or zero, given that $1-\Gamma_{0}\left(b_{s}\right) \geq 0$ and $\Gamma_{0}\left(b_{s}\right)-\Gamma_{1}\left(b_{s}\right)>0$ for all $b_{s}$. Because of the latter inequality, on the other hand, the second term on the right-hand side of Eq. (A.16) is strictly negative. Consequently, it is possible to solve the system (A.7)-(A.8) for every $\mathbf{k}$ and eventually express $\widetilde{\phi}$ and $\widetilde{B}_{\|}$in the form (A.1), (A.3), with the help of the representations (A.5), in the following way:

$$
\begin{aligned}
& \widetilde{\phi}(\mathbf{x}, t)=\sum_{\mathbf{k} \in \mathscr{D}} \widetilde{\phi}_{\mathbf{k}}(t) \mathrm{e}^{i \mathbf{k} \cdot \mathbf{x}}= \\
& =\sum_{\mathbf{k} \in \mathscr{D}} L_{\phi_{e \mathbf{k}}} \widetilde{g}_{e \mathbf{k}}\left(v_{\|}, \mu_{0 s}, t\right) \mathrm{e}^{i \mathbf{k} \cdot \mathbf{x}}+\sum_{\mathbf{k} \in \mathscr{D}} L_{\phi_{i \mathbf{k}}} \widetilde{g}_{i \mathbf{k}}\left(v_{\|}, \mu_{0 s}, t\right) \mathrm{e}^{i \mathbf{k} \cdot \mathbf{x}} \\
& \equiv L_{\phi_{e}} \widetilde{g}_{e}+L_{\phi_{i}} \widetilde{g}_{i}, \\
& \widetilde{B}_{\|}(\mathbf{x}, t)=\sum_{\mathbf{k} \in \mathscr{D}} \widetilde{B}_{\| \mathbf{k}}(t) \mathrm{e}^{i \mathbf{k} \cdot \mathbf{x}}= \\
& =\sum_{\mathbf{k} \in \mathscr{D}} L_{B_{e \mathbf{k}}} \widetilde{g}_{e \mathbf{k}}\left(v_{\|}, \mu_{0 s}, t\right) \mathrm{e}^{i \mathbf{k} \cdot \mathbf{x}}+\sum_{\mathbf{k} \in \mathscr{D}} L_{B_{i \mathbf{k}}} \widetilde{g}_{i \mathbf{k}}\left(v_{\|}, \mu_{0 s}, t\right) \mathrm{e}^{i \mathbf{k} \cdot \mathbf{x}} \\
& \equiv L_{B_{e}} \widetilde{g}_{e}+L_{B_{i}} \widetilde{g}_{i},
\end{aligned}
$$

with

$$
\begin{aligned}
L_{\phi_{s \mathbf{k}}} & =\frac{1}{\operatorname{det} \mathcal{Q}_{\mathbf{k}}}\left(N_{B \mathbf{k}} M_{s \mathbf{k}}-M_{B \mathbf{k}} N_{s \mathbf{k}}\right), \\
L_{B_{s \mathbf{k}}} & =\frac{1}{\operatorname{det} \mathcal{Q}_{\mathbf{k}}}\left(M_{\phi \mathbf{k}} N_{s \mathbf{k}}-N_{\phi \mathbf{k}} M_{s \mathbf{k}}\right)
\end{aligned}
$$


As far as Eq. (A.12) is concerned, solving with respect to $\widetilde{A}_{\| \mathbf{k}}$ is possible without imposing restrictions on $\widetilde{g}_{e}$ and $\widetilde{g}_{i}$, if $O_{A \mathbf{k}}$ never vanishes for all $\mathbf{k}$. If $\Theta_{s} \geq 1$ for $s=e, i$ then this condition is satisfied. This follows from the fact that $\Gamma_{0}\left(b_{s}\right)>0$, $1-\Gamma_{0}\left(b_{s}\right) \geq 0$ and $k_{\perp}^{2} \geq 0$ for all $\mathbf{k}$. If $\Theta_{e}$ or $\Theta_{i}$ are less than one, then a sufficient condition for invertibility can be found assuming that one of the species (say ions) has an isotropic equilibrium distribution $\left(\Theta_{i}=1\right)$, whereas electrons have parallel equilibrium temperature greater than the perpendicular equilibrium temperature (i.e. $\Theta_{e}<1$ ). In this case the quantity $O_{A \mathbf{k}}$ reads

$$
O_{A \mathbf{k}}=\sum_{s} \frac{q_{s}^{2} n_{0}}{m_{s} c} \Gamma_{0}\left(b_{s}\right)+\frac{c}{4 \pi} k_{\perp}^{2}+\frac{e^{2} n_{0}}{m_{e} c}\left(1-\frac{1}{\Theta_{e}}\right)\left(1-\Gamma_{0}\left(b_{e}\right)\right) .
$$

The sum of the first two terms on the right-hand side of Eq. (A.21) is positive, whereas the last term, which depends on the temperature anisotropy, is negative for $b_{e}>0$ and vanishes for $b_{e}=0$. If the absolute value of the latter term is sufficiently small, however, $O_{A \mathbf{k}}$ is always positive and Eq. (A.12) can be solved with respect to $\widetilde{A}_{\| \mathbf{k}}$ for every k. For $k_{\perp}^{2}=0$ this is always the case. For $k_{\perp}^{2}>0$, the condition $O_{A \mathbf{k}}>0$ can be rewritten as

$$
\frac{1}{\Theta_{e}}<1+\frac{\delta^{2} \Gamma_{0}\left(b_{i}\right)+\Gamma_{0}\left(b_{e}\right)+k_{\perp}^{2} d_{e}^{2}}{1-\Gamma_{0}\left(b_{e}\right)} \equiv \Psi\left(k_{\perp}^{2}\right),
$$

where $d_{e}=c\left(m_{e} /\left(4 \pi e^{2} n_{0}\right)\right)^{1 / 2}$ is the electron skin depth. For $k_{\perp}^{2}>0$ the function $\Psi$ is continuous, takes always values greater than 1 and, in particular, it is bounded from below by an infimum taken at a value of $k_{\perp}^{2}$ that we denote with $k_{i n f}^{2}$. Therefore, if the condition

$$
1>\Theta_{e}>\frac{1}{\Psi\left(k_{i n f}^{2}\right)}
$$

is satisfied, then the condition (A.22) is satisfied for every $\mathbf{k}$ and the magnetic potential $\widetilde{A}_{\|}$is well defined. 
More explicitly, we can write

$$
\begin{aligned}
& \widetilde{A}_{\|}(\mathbf{x}, t)=\sum_{\mathbf{k} \in \mathscr{D}} \widetilde{A}_{\| \mathbf{k}}(t) \mathrm{e}^{i \mathbf{k} \cdot \mathbf{x}}= \\
& =\sum_{\mathbf{k} \in \mathscr{D}} L_{A_{e \mathbf{k}}} \widetilde{g}_{e \mathbf{k}}\left(v_{\|}, \mu_{0 s}, t\right) \mathrm{e}^{i \mathbf{k} \cdot \mathbf{x}}+\sum_{\mathbf{k} \in \mathscr{D}} L_{A_{i \mathbf{k}}} \widetilde{g}_{i \mathbf{k}}\left(v_{\|}, \mu_{0 s}, t\right) \mathrm{e}^{i \mathbf{k} \cdot \mathbf{x}} \\
& \equiv L_{A_{e}} \widetilde{g}_{e}+L_{A_{i}} \widetilde{g}_{i},
\end{aligned}
$$

where

$$
L_{A_{s \mathbf{k}}}=\frac{O_{s \mathbf{k}}}{O_{A \mathbf{k}}}
$$

If, on the other hand, for $\Theta_{e}<1$ and/or $\Theta_{i}<1$, there exist some wave vector $\overline{\mathbf{k}}$ such that $O_{A \overline{\mathbf{k}}}=0$, then, for Eq. (A.12) to hold, we require that $\widetilde{g}_{e \overline{\mathbf{k}}}=\widetilde{g}_{i \overline{\mathbf{k}}}=0$, which imposes some restrictions on the generalized distribution functions $\widetilde{g}_{e}$ and $\widetilde{g}_{i}$.

By a similar argument is is also possible to find conditions for the magnetic potential appearing in Eq. (43).

Given the expressions (A.17), (A.18) and (A.24) for the operators $L_{\phi_{s}}, L_{B_{s}}$ and $L_{A_{s}}$ and the definition (23) for the operators $\mathcal{J}_{i_{s s^{\prime}}}$, the property (22) follows easily.

\section{References}

[1] Brizard A and Hahm T S 2007 Rev. Mod. Phys. 79421

[2] Howes G G, Cowley S C, Dorland W, Hammett G W, Quataert E and Schekochihin A A 2006 The Astrophysical Journal 651 590-614

[3] Kunz W M, Schekochihin A A, Chen C H K, Abel I G and Cowley S C 2015 J. Plasma Phys. 81 325810501

[4] Brizard A 1992 Phys. Fluids 4 1213-1228

[5] Dorland W and Hammett G W 1993 Phys. Fluids B 5 812-835

[6] Hammett G W, Dorland W and Perkins F W 1992 Phys. Fluids B 42052

[7] Beer M A and Hammett G W 1996 Phys. Plasmas 34046

[8] Hammett G W, Beer M A, Dorland W, Cowley S C and Smith S A 1993 Plasma Phys. Control. Fusion 35973

[9] Snyder P B and Hammett G W 2001 Phys. Plasmas 8 3199-3216

[10] Strintzi D, Scott B and Brizard A 2005 Phys. Plasmas 12052517

[11] Scott B 2010 Phys. Plasmas 17102306

[12] Madsen J 2013 Phys. Plasmas 20072301

[13] Waelbroeck F L, Hazeltine R D and Morrison P J 2009 Phys. Plasmas 16032109

[14] Waelbroeck F and Tassi E 2012 Commun. Nonlinear Sci. Numer. Simulat. 172171

[15] Grasso D and Tassi E 2015 J. Plasma Phys. 81495810501

[16] Keramidas Charidakos I, Waelbroeck F L and Morrison P J 2015 Phys. Plasmas 22112113

[17] Grasso D, Tassi E and Waelbroeck F L 2010 Phys. Plasmas 17082312

[18] Comisso L, Grasso D, Tassi E and Waelbroeck F L 2012 Phys. Plasmas 19042103 
[19] Despain K M 2011 Gyrofluid modeling of turbulent, kinetic physics Ph.D. thesis University of Maryland

[20] Tassi E 2015 Annals of Physics 362239

[21] Tassi E 2017 Eur. Phys. J. D 71269

[22] Morrison P J 1998 Rev. Mod. Phys. 70 467-521

[23] Marsden J E and Ratiu T S 2002 Introduction to Mechanics and Symmetry (Springer-Verlag, Berlin)

[24] de Blank H 2001 Phys. Plasmas 83927

[25] Tassi E 2014 Eur. Phys. J. D 68196

[26] Tassi E 2014 J. Phys. A: Math. Theor. 47195501

[27] Szegö G 1975 Othogonal Polynomials (Amer. Math. Soc., Providence, Rhode Island)

[28] Gillis J and Weiss G 1960 Mathematics of Computation 1460

[29] Zocco A and Schekochihin A 2011 Phys. Plasmas 18102309

[30] Schep T J, Pegoraro F and Kuvshinov B N 1994 Phys. Plasmas 1 2843-2851

[31] Passot T, Sulem P L and Tassi E 2018 Phys. Plasmas 25042107

[32] Hasegawa A 1975 Plasma Instabilities and Nonlinear Effects (Springer-Verlag)

[33] Kunz W M, Abel I G, Klein K G and Schekochihin A A 2018 J. Plasma Phys. 84715840201

[34] Perin M, Chandre C and Tassi E 2016 J. Phys. A: Math. Theor. 49305501

[35] Tassi E 2016 Theor. and Math. Phys. 1881377

[36] Tassi E, Sulem P L and Passot T 2016 J. Plasma Phys. 82705820601

[37] Tassi E, Grasso D, Borgogno D, Passot T and Sulem P L 2018 Journal of Plasma Physics 84 725840401 